\title{
Interspecific differences, plastic, and evolutionary responses to a heat wave in three co-occurring Daphnia species
}

\author{
Héléne Vanvelk $\mathbb{( D}^{1}{ }^{1 *}$ Lynn Govaert, ${ }^{2,3,4}$ Edwin M. van den Berg, ${ }^{1}$ Kristien I. Brans, ${ }^{1}$ Luc De Meester ${ }^{1,5,6}$ \\ ${ }^{1}$ Laboratory of Aquatic Ecology, Evolution and Conservation, KU Leuven, Leuven, Belgium \\ ${ }^{2}$ Department of Evolutionary Biology and Environmental Studies, University of Zurich, Zürich, Switzerland \\ ${ }^{3}$ Department of Aquatic Ecology, Eawag: Swiss Federal Institute of Aquatic Science and Technology, Dübendorf, Switzerland \\ ${ }^{4}$ URPP Global Change and Biodiversity, University of Zurich, Zurich, Switzerland \\ ${ }^{5}$ Leibniz Institute für Gewasserökologie und Binnenfischerei (IGB), Berlin, Germany \\ ${ }^{6}$ Institute of Biology, Freie Universität Berlin, Berlin, Germany
}

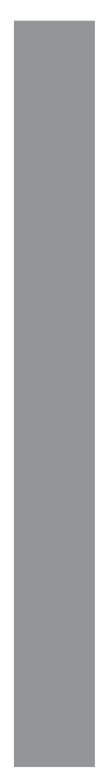

\begin{abstract}
Many studies document genetic and phenotypic trait changes of species in response to climate change, or document how evolution of individual species can impact population abundances and community composition. An integration of population and community-level responses requires, however, a multiple species approach. Here we quantify among- and within-species differences in thermal tolerance and life-history traits in three co-occurring Daphnia species upon exposure to a naturally occurring heat wave. Populations of randomly isolated clones of Daphnia magna, Daphnia pulicaria, and Daphnia galeata from the same pond were exposed to a natural heat wave in outdoor mesocosms. We subsequently conducted a common garden experiment in the laboratory using clonal lineages isolated at the end of the mesocosm selection experiment, at two rearing temperatures, measuring thermal tolerance and life-history traits. We find pronounced plasticity responses to higher rearing thermal regime in each study species. We observe only few significant microevolutionary responses involving evolution of plasticity in D. pulicaria. Yet in terms of effect size, evolutionary trait change within species contributes more than $25 \%$ to total trait change in response to the heat wave for a majority of the trait $\times$ species combinations. The relative importance of intraspecific to interspecific variation varies widely among traits. Our results show that the relative importance of interspecific variation, phenotypic plasticity, and evolutionary trait change differs strongly depending on the set of species and traits studied. Taking into account this variation at different levels of biological organization is important to predict community-wide responses to global change.
\end{abstract}

Climate change is profoundly affecting biota on a global scale, impacting ecosystems and their functioning worldwide (IPCC 2014). The impact of climate change is manifested at all biological levels, ranging from genetic changes, changes in physiology, morphology, behavior, and life-history traits of populations, to alterations in populations dynamics, changes in community structure and biodiversity, and changes in

*Correspondence: helene.vanvelk@kuleuven.be

Additional Supporting Information may be found in the online version of this article.

Author Contributions Statement: H.V., L.G., and L.D.M. designed the study. E.M.v.d.B. isolated the animals, cultured the lineages and carried out the selection experiment, with guidance from K.I.B. and L.D.M. H.V. carried out the heat tolerance and life table common garden experiment with input from K.I.B. and L.D.M. Data analysis was done by H.V. with suggestions by L.G., K.I.B., and L.D.M. H.V. wrote the $1^{\text {st }}$ version of the manuscript with input of L.D.M. and L.G. All authors worked on the subsequent versions of the manuscript. ecosystem functioning and structure (Scheffers et al. 2016). These changes at different levels of biological organization can interact with each other, which leads to complex feedback loops that challenge our capacity to make reliable predictions on the ecological consequences of climate change (Urban et al. 2016).

Species can respond to climate change through migration or trait change mediated by phenotypic plasticity (the capacity of a single genotype to produce different phenotypes; DeWitt et al. 1998) or genetic changes (evolution; Gienapp et al. 2008). Phenotypic plasticity of a trait can also evolve itself (Pfennig et al. 2010). Adaptation in response to climate change can involve genetic changes in multiple traits, including phenology (Parmesan and Yohe 2003), life-history traits (Vindenes et al. 2014), and thermal tolerance (Carvalho 1987; Geerts et al. 2015). While multiple studies have documented important trait changes in species upon climate change both in natural (Sheridan and Bickford 2011) as well as in 
laboratory settings (Neuwald and Valenzuela 2011), only a minor subset of them are designed to allow quantification of the relative importance of phenotypic plasticity vs. evolutionary trait change, for example, by carrying out transplant or common garden experiments (Merilä and Hendry 2013).

Warming is one of the key components of climate change. In addition to an increase in average temperature, extreme events such as heat waves are occurring more frequently in a warming climate (IPCC 2014). Minor changes in average temperature can lead to disproportionally large effects in intensity and frequency of heat waves (Perkins 2015). It has been estimated that the likelihood of occurrence of a heat wave such as the one in Europe in the summer of 2018 has increased due to anthropogenic climate change (IPCC 2014). By 2040, heat waves are expected to become common (Stott et al. 2004). There are an increasing number of studies that quantify ecological and evolutionary responses to heat waves, both in nature as well as in laboratory experiments (Ciais et al. 2005; Reichstein et al. 2007). These studies, however, mostly focus on single species responses.

Warming has been shown to profoundly change the characteristics and functioning of ponds and shallow lakes. For example, warming increases the likelihood of the occurrence of toxic cyanobacteria blooms and exacerbates the effects of eutrophication (Kosten et al. 2012). Climate warming also profoundly affects food web structure in lakes and ponds (Winder and Schindler 2004), among others because higher temperatures often result in body size reduction in ectothermic species (Daufresne et al. 2009) in accordance with the temperature-size rule (Atkinson 1994). In addition to direct effects of increasing temperatures on species traits, climate warming is also predicted to impact biota through a multitude of indirect effects, for example, through a change in food supply (e.g., abundance and quality of phytoplankton) or changes in the strength of interspecific interactions such as competition and predation (Moore 1996; Jeppesen et al. 2010).

Species from the water flea genus Daphnia are keystone species in ponds and shallow lakes (Lampert 2006). They control phytoplankton dynamics and are an important food source for planktivorous fish (Miner et al. 2012). They have been shown to respond to warming through phenotypic plasticity as well as through evolutionary responses. Studies using space-for-time substitutions (Mitchell and Lampert 2000), experimental evolution methods (Van Doorslaer et al. 2010), and laboratory exposures (Paul et al. 2004) have revealed that these adaptations involve several traits, including thermal tolerance (Yampolsky et al. 2014; Geerts et al. 2015), body size (Geerts et al. 2015), concentration of heat-shock proteins (Paul et al. 2004) and hemoglobin (Zeis et al. 2013), and changes in somatic growth rate and intrinsic population growth rate (Van Doorslaer et al. 2010; Herrmann et al. 2017). Due to their central position in the food web, trait changes in Daphnia populations have the potential to affect community composition and ecosystem structure of ponds and lakes (De Meester et al. 2011; Pantel et al. 2015).

Most studies on evolutionary responses to climate warming focus on single species. Yet, to achieve a better understanding of evolutionary responses and their impact on community ecology, there is a need to obtain data on evolutionary responses of multiple coexisting species (Govaert et al. 2016; De Meester et al. 2019). Different Daphnia species have largely overlapping niches (Ranta et al. 1993) and are frequently found to co-occur in natural communities (Altermatt et al. 2008). As temperature effects are expected to be species specific, depending among others on their thermal tolerance ranges and amount of genetic variation (WojtalFrankiewicz 2012), divergence in thermal responses are expected to alter interspecific interactions (Huey et al. 2012). Studies that simultaneously quantify plastic and evolutionary trait responses to warming in multiple coexisting species are, however, missing.

In this study, we quantified whether and how three coexisting Daphnia species respond to a naturally occurring heat wave. Specifically, we aimed to: (1) test whether and to what extent each species shows evolutionary trait change in response to exposure to a heat wave; (2) quantify for each species the relative importance of plasticity, constitutive evolution and evolution of plasticity to total trait change in response to a temperature increase; and (3) compare intraspecific plastic and evolutionary trait variation to interspecific differences in life-history traits and thermal tolerance. To this end, we hatched multiple clones from three co-occurring Daphnia species from the egg bank of a natural pond, cultured the resulting clonal lineages for multiple generations under standardized laboratory conditions to purge them from maternal effects, and then inoculated genetically variable but standardized populations of each of the three species separately in mesocosms. Here we took advantage of a naturally occurring heat wave to quantify evolutionary responses to warming in all three species by comparing isolates from the mesocosms following the heat wave to clones isolated from the original population in a follow-up common garden experiment. In this common garden experiment, we quantified heat tolerance and life-history traits at two different temperatures to be able to quantify phenotypic plasticity, evolution of mean trait value, and evolution of phenotypic plasticity in each of the three co-occurring species.

\section{Material and methods}

\section{Isolation of multiple clonal lineages per species}

Dormant cladoceran eggs were hatched from the upper $2 \mathrm{~cm}$ soil layer of Langerodevijver (Belgium, 50 49'42.2' $\mathrm{N}-$ $4^{\circ} 38^{\prime} 23.7^{\prime \prime} \mathrm{E}$ ), a shallow eutrophic pond, in April-May 2018. This pond is known to have a cladoceran community that is dominated by three Daphnia species: Daphnia magna, Daphnia pulicaria, and Daphnia galeata. While there are other 
cladoceran species present in the pond (e.g., Ceriodaphnia quadrangula, Simocephalus vetulus, Scapholeberis mucronata, Chydorus sphaericus), the three Daphnia species usually make up $>85 \%$ of the cladoceran community biomass during spring (E. M. van den Berg unpubl. observ.). Hatching of the dormant eggs was achieved by spreading the sediment in thin layers in white trays in dechlorinated tap water at $20^{\circ} \mathrm{C}$ and under a $16: 8$-h light: dark photoperiod in the laboratory. The trays were screened for hatchlings every 2 days. A total of 70 clones from each of the three Daphnia species was isolated and grown in individual $210-\mathrm{mL}$ jars to start clonal cultures. Culturing was done in dechlorinated tap water, under a 16 : 8-h light : dark photoperiod and at $20^{\circ} \mathrm{C}$. The animals were fed $1 \times 10^{5}$ cells/mL of the unicellular algae Acutodesmus obliquus twice a week, and $80 \%$ of the medium was refreshed once a week. As dormant eggs result from sexual reproduction, each hatchling represents a distinct genotype. Because reproduction in the laboratory is through amictic parthenogenesis in these cyclically parthenogenetic species, isolating offspring of different dormant eggs and using these to start separate cultures under favorable conditions yields cultures of genetically identical animals (i.e., clones) that represent different genotypes among cultures. In this way, we obtained a representative sample of the genetic diversity of each of the populations of the three co-occurring species. The generation time of our study species ranges between approximately 8-11 days at $20^{\circ} \mathrm{C}$ and $6-8$ days at $26^{\circ} \mathrm{C}$, with the larger species having a longer generation time than the smaller ones (Bottrell et al. 1976; Korpelainen 1986; Vanvelk H. data life table experiment).

\section{Selection experiment}

From mid-June to the end of July 2018, we performed an outdoor mesocosm selection experiment in our outdoor mesocosm area (Belgium, 50 51'37.2 ${ }^{\prime \prime} \mathrm{N}-4^{\circ} 40^{\prime} 46.2^{\prime \prime} \mathrm{E}$ ). Monospecific populations of D. magna, D. pulicaria, and D. galeata were exposed to a naturally occurring heat wave (schematic overview Fig. 1). D. magna populations were seeded with 69 clones, each represented by two 5-day-old individuals, D. pulicaria populations consisted of 67 clones, each represented by six 6-day-old individuals, and the populations of D. galeata consisted of 64 clones, each represented by six 5-day-old individuals. One of the 64 clones used to start the D. galeata populations appeared to have been accidently switched with a $D$. pulicaria clone. We did not find $D$. pulicaria in any of the counts of these populations nor in the clonal lineages we isolated from these populations at the end of the selection experiment, but at least at the start there was some contamination in the D. galeata populations $\left(1 / 64^{\text {th }}\right.$ of the inoculum). For each species, all clones were cultured for more than four generations under standardized laboratory conditions (see above) to obviate inference from (great)(grand)maternal effects, after which they were inoculated to 200-L mesocosms. We inoculated a different amount of individuals per mesocosm for the different species (more individuals per clone for the smaller species $D$. pulicaria and D. galeata than for the larger species D. magna) so that all populations, irrespective of species, would more or less start the selection phase with a similar biomass.

The animals were inoculated on 15 June 2018 and were allowed to grow and form populations for approximately 6 weeks before we isolated individuals on 01 August 2018. Prior to inoculation of the Daphnia, the mesocosms were filled with $160 \mathrm{~L}$ dechlorinated tap water and $10 \mathrm{~L}$ filtered Langerodevijver pond water (filtered over a $64-\mu \mathrm{m}$ mesh to remove all zooplankton) to inoculate a natural bacterioplankton and phytoplankton community. Nutrients $(4.12 \mathrm{~g}$ of $\mathrm{NaNO}_{3}$ and $0.37 \mathrm{~g}$ of $\mathrm{KH}_{2} \mathrm{PO}_{4}$ ) and an inoculum of laboratory grown $A$. obliquus alga $\left(85 \times 10^{8}\right.$ cells) were added as well. There were five replicate mesocosms for each species, resulting into a total of 15 mesocosms per temperature treatment. The original design involved two treatments: an ambient and a heated $\left(+4^{\circ} \mathrm{C}\right)$ treatment. The summer of 2018 had, however, the highest average air temperature since the beginning of systematic measurements (KMI 2018), with an average maximum temperature of $24.5^{\circ} \mathrm{C}$ (Supporting Information; KMI 2018) and the occurrence of a pronounced natural heat wave (i.e., air temperature exceeding $25^{\circ} \mathrm{C}$ for a minimum of $5 \mathrm{~d}$, with minimal 3 days that exceed $30^{\circ} \mathrm{C}$ ) during the period of $13 / 7$ to $28 / 7$. Before and after this period, two additional heat spikes happened where the temperature exceeded $25^{\circ} \mathrm{C}$, from $25 / 6$ to $08 / 7$ and $29 / 7$ to $31 / 7$ (KMI 2018). This prolonged period of anomalously warm air temperatures affected the water temperature in the mesocosms, resulting in 22 days during which the average day temperature of the water in our ambient mesocosms exceeded $25^{\circ} \mathrm{C}$ (Fig. S1). These warm days were distributed into two heat waves of 10 and 9 days and heat spikes of 4 days. As a result of the pronounced heat wave during the experiment, nearly all populations in the $+4^{\circ} \mathrm{C}$ heated mesocosms went extinct (except the D. magna populations and one $D$. pulicaria population). Therefore, we could not study anymore the response to a controlled heating treatment across the three species. However, we could capitalize on the naturally occurring heat wave to study how this impacted the evolution of the three Daphnia populations in the ambient treatment mesocosms, by comparing the populations from the mesocosm experiment to the original populations hatched from the dormant egg bank of Langerodevijver.

After 6 weeks, we randomly isolated six adult females from each mesocosm, resulting in 30 isolated clonal lineages for each species. These clonal lineages were kept under standardized laboratory conditions $\left(23^{\circ} \mathrm{C}, 14: 10\right.$ light : dark photoperiod, fed $5 \times 10^{4}$ cells $/ \mathrm{mL}$ A. obliquus daily, medium refreshed $50 \%$ twice per week) for at least $2-3$ generations to minimize interference from maternal effects. In this way, we established 30 ( 6 clonal lineages $\times 5$ replicated mesocosms) clonal lineages of each species from populations that were exposed to selection linked to a natural heat wave (schematic overview Fig. 1). 


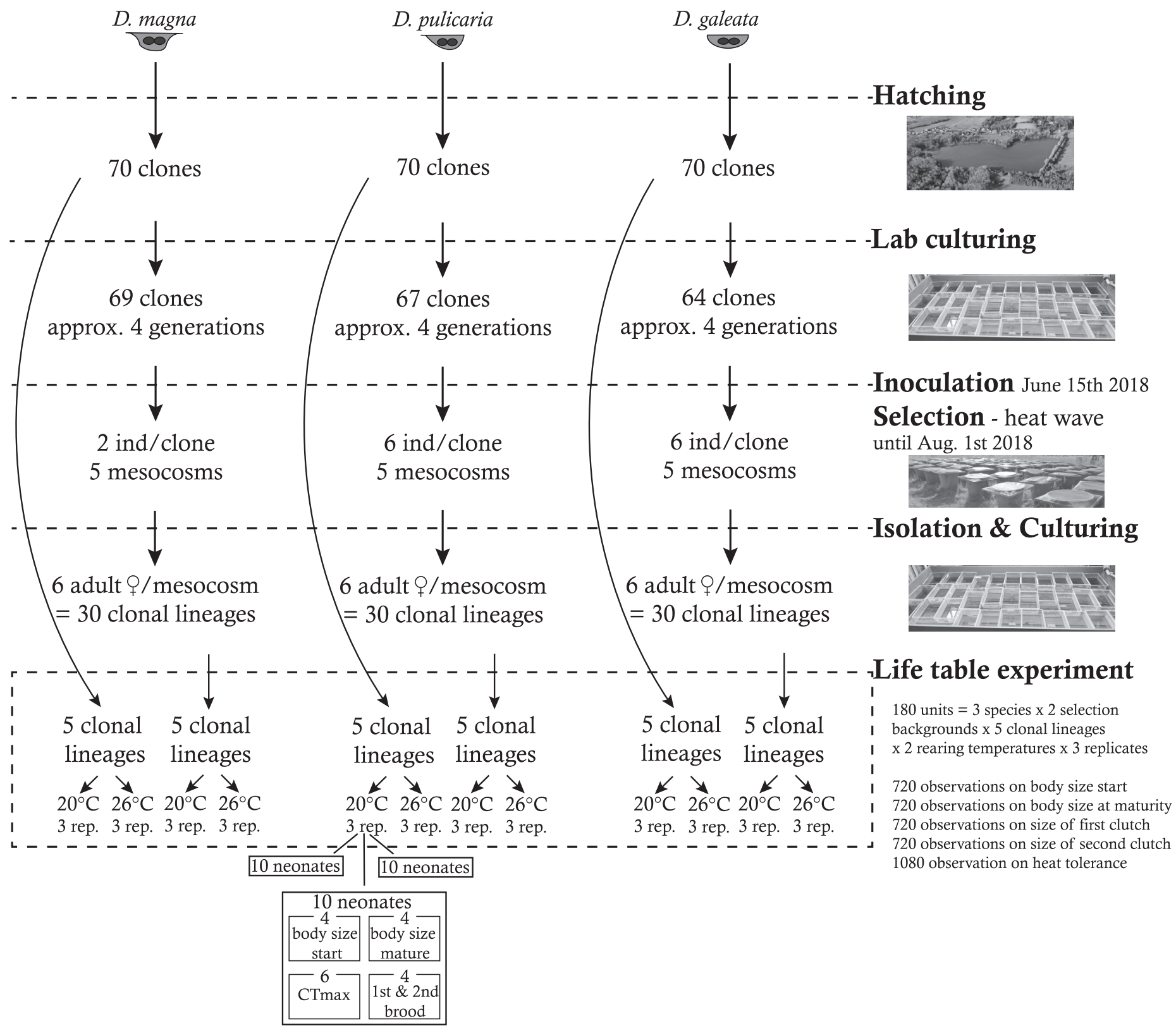

Fig 1. Schematic overview of the design of our study. We hatched animals of three species from the natural egg bank of a single pond. After purging for maternal effects by several generations of culture in the laboratory, we used a subset of clones to initiate a mesocosm experiment in which the populations were exposed to a natural heat wave. After this selection, we isolated clonal lineages from the different mesocosms and cultured them in the laboratory for several generations. Then we carried out a life table and heat tolerance experiment using five clonal lineages from both the original populations and the mesocosm populations of each species, at two different temperatures.

We here denote the isolates as clonal lineages, as we used the isolated females from the mesocosms to start clonal cultures. Yet, while we know that all individuals within a lineage are genetically identical, we are not absolutely sure that the clonal lineages are all genetically distinct from each other, as there has been clonal reproduction in the mesocosms. As a result, different clonal lineages may potentially belong to the same clone isolated from the original population.

\section{Life table experiment and response variables}

We performed a full-factorial life table experiment using, for each species, a subset of 5 clonal lineages from the total set of 30 clonal lineages isolated from the heat wave-exposed mesocosms. We attempted to choose clonal lineages from as many replicate mesocosms as possible, and within that constraint randomly picked five clones. For D. magna this resulted in one randomly selected clonal lineage per mesocosm, for 
D. pulicaria the five clonal lineages were derived from three replicate mesocosms, and for $D$. galeata, they originated from two replicate mesocosms. For each of the three species, we also included five randomly chosen clones of the original population directly hatched from Langerodevijver. Experimental animals were reared at two temperatures: $20^{\circ} \mathrm{C}$ and $26^{\circ} \mathrm{C}$. The common garden experiment thus involved 3 species $\times 2$ selection backgrounds (heat wave-exposed vs. nonexposed original populations $) \times 5$ clones $\times 2$ culture temperatures $\times 3$ replicates $=180$ experimental units (schematic overview Fig. 1). The set-up of this common garden experiment allows to test for both phenotypic plasticity when the animals are reared under warming conditions in the laboratory $\left(26^{\circ} \mathrm{C}\right.$ vs. $\left.20^{\circ} \mathrm{C}\right)$ as well as to quantify the evolutionary response following exposure to a heat wave in the mesocosms. The comparison of clonal lineages isolated from mesocosm populations that experienced a pronounced heat wave with clones that were directly hatched from the dormant egg bank in nature tests for evolutionary responses in the mesocosm experiment and might involve both a response to the heat wave and to the mesocosm conditions that likely differed in important aspects from the conditions in Langerodevijver (e.g., mesocosms started from low population densities, only one species present, no predation, ...).

At the start of the life table experiment, 500-mL jars containing dechlorinated (24-h aged) tap water were inoculated with $102^{\text {nd }}$-brood female neonates $(<24 \mathrm{~h}$ old $)$. These jars were randomly allocated to a position in one of two water baths that were kept at either $20^{\circ} \mathrm{C}$ or $26^{\circ} \mathrm{C}$. The temperatures used were chosen to represent a typical vs. high summer temperature in the region (Langerodevijver, Belgium), and are both reasonably close to the optimal temperature for D. magna growth (Mitchell and Lampert 2000). Photoperiod was $14: 10$-h light : dark. The medium in the cultures was refreshed every other day with 30\% new medium, and food concentration in all cultures was daily restored by adding $4 \times 10^{4}$ cells of $A$. obliquus algae (approximately 0.8-1 mg C/L).

We quantified heat tolerance ( $\mathrm{CT}_{\max }$; Huey et al. 1992; Geerts et al. 2015) as this trait is expected to be directly linked to the ability of animals to cope with a heat wave. We also quantified life history traits age at maturity and clutch size as they are directly related to population growth rate, and body size. Body size is a key trait structuring life history strategies and occurrence patterns in zooplankton (Tessier et al. 2000; Gianuca et al. 2016b) and is known to be associated with heat tolerance (Geerts et al. 2015; Brans et al. 2017b). All measured traits are known to respond to changes in temperature (Mitchell and Lampert 2000; Van Doorslaer et al. 2010; Geerts et al. 2015). All cohorts were monitored daily. Neonate body size (i.e., length from top of eye to base of tail spine) was measured for 4 of the 10 neonates that were inoculated at the start of the experiment (stereomicroscope Olympus SZX12). Size at maturity was measured as the average body size of four randomly chosen adults upon reaching maturity (i.e., $1^{\text {st }}$ brood in the brood pouch). Upon reaching maturity, we randomly chose six adult individuals to determine critical maximum temperature $\left(\mathrm{CT}_{\max }\right) . \mathrm{CT}_{\max }$ was determined following Geerts et al. (2015) and Brans et al. (2017b): a single animal is placed in an Eppendorf tube filled with water, which is exposed to a constant heating of $1^{\circ} \mathrm{C}$ per $20 \mathrm{~s}$ using a thermoblock; the water temperature at which the animal loses all motoric function is recorded as $\mathrm{CT}_{\max }$. For the remaining four adults in the jar, we quantified the average clutch size of the $1^{\text {st }}$ and $2^{\text {nd }}$ brood as well as the days of clutch release. Thanks to the fact that the cohorts were sufficiently well synchronized, it was possible to differentiate between the $1^{\text {st }}$ and $2^{\text {nd }}$ clutch in all culture jars by checking daily for offspring. For all traits, we thus obtained average value per replicate of each species $\times$ selection background $\times$ clone $\times$ rearing temperature combination. From the measured life-history traits, we additionally calculated daily somatic growth rate, as $G j=(\ln ($ size at maturity) - $\ln ($ size at birth))/time to maturation in days). The number of offspring combined with their time of birth allows the calculation of performance $r$, as the intrinsic population growth under assumption of no mortality, by solving the Lotka-Euler equation (Kot 2001) using the "uniroot" function in R from the "stats" package (R Core Team 2013).

\section{Statistical analyses \\ Effects of species, selection, and rearing temperature}

We performed a multivariate analysis of variance (MANOVA) on the total set of traits $\left(\mathrm{CT}_{\max }\right.$, size at maturity, age at maturation, clutch size of $1^{\text {st }}$ and $2^{\text {nd }}$ brood, somatic growth rate and performance $r$ as response variables), with species, temperature during the life table experiment and selection background (heat wave exposed vs. original) as fixed effects, and clone (nested within selection background) and day of measurement as two random factors. Data were standardized to a mean of 0 and a standard deviation of 1 . For all traits we used the average value per replicate in the data analysis. The MANOVA on the trait data of all species together, and subsequently for each species separately, allows testing for overall differences among species as well as among background and temperature for each species separately. We then used Type II ANOVAs on each trait separately to detect trait-specific responses (Fox and Weisberg 2019). Again, tests were first run for all species together, and subsequently for each species separately. For the model that had $\mathrm{CT}_{\max }$ as a response variable, we also included size at maturity as a covariate, as it is known that $\mathrm{CT}_{\max }$ values often depend on body size (Geerts et al. 2015). Afterward, post hoc testing for differences between species pairs was done using lsmeans function (Lenth 2016). Model assumptions such as normality of residuals and homogeneity of variance were checked and formally tested with the Shapiro-Wilk test and the Levene test, respectively, for each statistical model and were met (Fox and Weisberg 2019; R Core Team 2013). To visualize which traits 
or trait combinations display the largest differences among species, selection background, and rearing temperature, we performed a principal components analysis (PCA; Legendre and Legendre 2012).

\section{Contribution of plasticity and evolution to trait change}

During a heat wave, the observed trait values in a population can change both due to evolution and phenotypic plasticity, given that animals from the original population growing at typical summer temperatures (approximately $20^{\circ} \mathrm{C}$ ) are replaced by animals of the exposed (and potentially evolved) population growing at a higher temperature. Assuming that the animals during the heat wave were exposed to approximately $26^{\circ} \mathrm{C}$, we can use the laboratory data of the original and exposed populations for each species to implement eco-evolutionary partitioning metrics such as the reaction norm approach (Govaert et al. 2016; Stoks et al. 2016). The reaction norm approach is an asymmetric version of the Geber method developed by Hairston Jr. et al. (2005) and Ellner et al. (2011). It deviates from classical genotype $\times$ environment variation partitioning because reaction norms are here evaluated at the population level, rather than at the genotype level. The reaction norm approach allows to quantify the absolute and relative contributions of (ancestral) plasticity, evolution of mean trait values (constitutive evolution) and evolution of plasticity to total population trait change expected during the heat wave. More specifically, it allows the calculation of the amount of each contributing factor (i.e., "effect size") to the total trait change. Ancestral plasticity (i.e., the plastic response of the original population) was calculated as the mean trait shift from the low $\left(20^{\circ} \mathrm{C}\right)$ to the high $\left(26^{\circ} \mathrm{C}\right)$ temperature treatment of the original population as observed in our laboratory common garden experiment. Constitutive evolution reflects the genetic shift in trait mean under original environmental conditions (i.e., $20^{\circ} \mathrm{C}$ ) and is calculated as the difference in trait mean between the heat wave-exposed and nonexposed original population in the low temperature condition. Evolution of plasticity (i.e., how the plasticity of a trait itself evolved over time) is calculated as the difference in the plastic response of the heat wave-exposed and the nonexposed original population. These three components add up to the total trait change (Govaert et al. 2016) that is expected to be observed in the field under the assumption that temperature is the key factor influencing the traits under study. The relative contribution of each of these components is then calculated as the components' absolute value divided by the sum of all components' absolute value. We applied this method to the univariate as well as multivariate trait change for each species separately using the trait values scaled across traits and species (scaling around mean of 0 with standard deviation of 1 ). The contributions of plasticity, constitutive evolution, and evolution of plasticity in case of multivariate trait change are calculated as Euclidean distances in multivariate trait space integrating all traits in a seven-dimensional vector (see Stoks et al. 2016 for details). We used the output of the PCA to visualize the multivariate reaction norms.

\section{Scaling intraspecific to interspecific differences}

The relative importance of intraspecific vs. interspecific variation for each trait was quantified by taking the variance component (i.e., regression sum of squares) of the previously described ANOVA model including all three species. Specifically, the variation explained by the predictor variable "species" estimates the importance of interspecific variation, while the variation explained by the predictor variable "selection background" and "rearing temperature" and interactions between these main effects as well as with the "species" main effect estimate together the importance of intraspecific variation.

To assess the extent to which intraspecific trait change in response to a heat wave alters interspecific differences, and how this relates to contributions of plasticity, mean trait evolution and evolution of plasticity, we partitioned and visualized the total trait change within each species using the reaction norm approach as described earlier, but on the nonstandardized data. Specifically, we used the species mean trait value of the original population at $20^{\circ} \mathrm{C}$ as a baseline to which absolute contributions of plasticity, mean trait evolution and evolution of plasticity were plotted. This allows to visualize absolute contributions to trait changes, while clearly reflecting how trait changes within species relate to the differences in trait values among species.

We then use these intraspecific and interspecific differences explicitly to scale the total amount of intraspecific trait change to the amplitude of interspecific differences at both $20^{\circ} \mathrm{C}$ and $26^{\circ} \mathrm{C}$. First, we calculate interspecific differences before warming (original population at $20^{\circ} \mathrm{C}$ ) and during the heat wave (evolved population at $26^{\circ} \mathrm{C}$ ) for each species pair. Interspecific differences at $20^{\circ} \mathrm{C}$ were calculated as the difference between average trait values of the nonexposed original population of each species in a particular species pair (i.e., D. magna-D. pulicaria, D. magna-D. galeata, and D. pulicaria-D. galeata). The interspecific difference at $26^{\circ} \mathrm{C}$ was calculated similarly, but instead using the average trait value of the heat wave-exposed population per species. Second, for each trait and original or exposed population of a species, we calculated intraspecific variation as the difference in mean trait values between the two most differentiated clones. This was done using data on clonal reaction norms per species (see Supporting information). Note that by taking the difference between the most differentiated clones within each population we tried to capture the range of intraspecific trait variation present in the population. By using clonal means, we do not use the most extreme values observed in our data set, but using the extreme values would make our analysis prone to chance effects. 


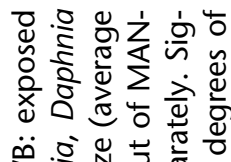

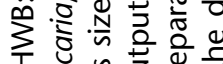

行 论

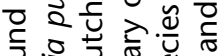

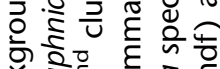

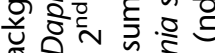

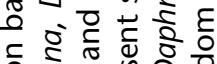

응 혼

政

बू. ำ

흥ㅎㅇ

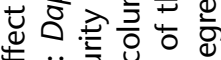

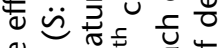

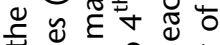

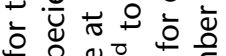

은. 은

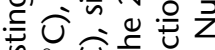

过过

정

ธ

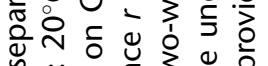

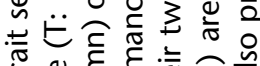

可语

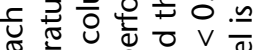

ब्र

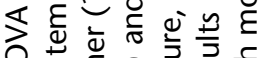

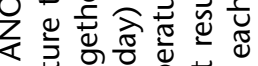

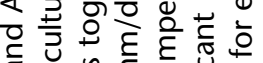

厄

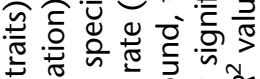

4。

造

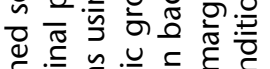

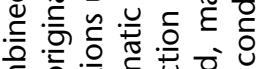

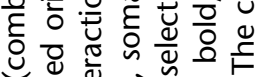

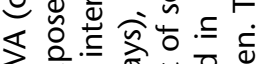

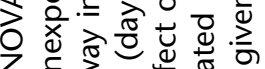

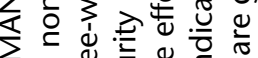

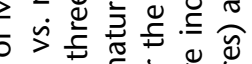

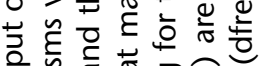

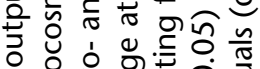

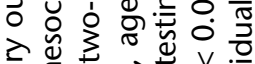

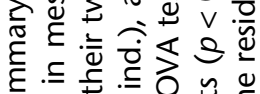

今

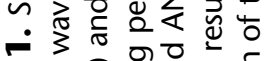

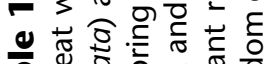

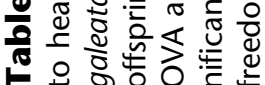

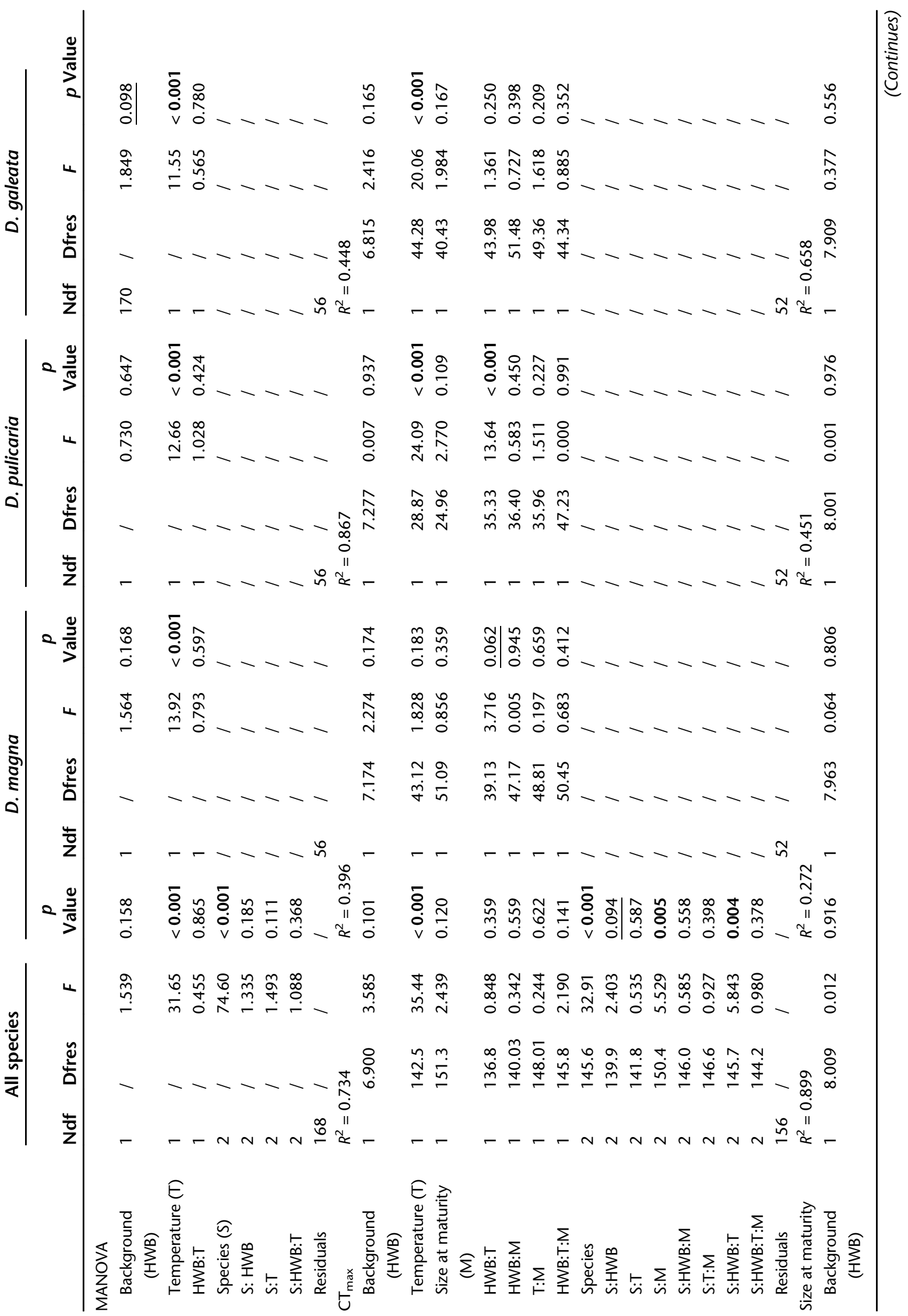




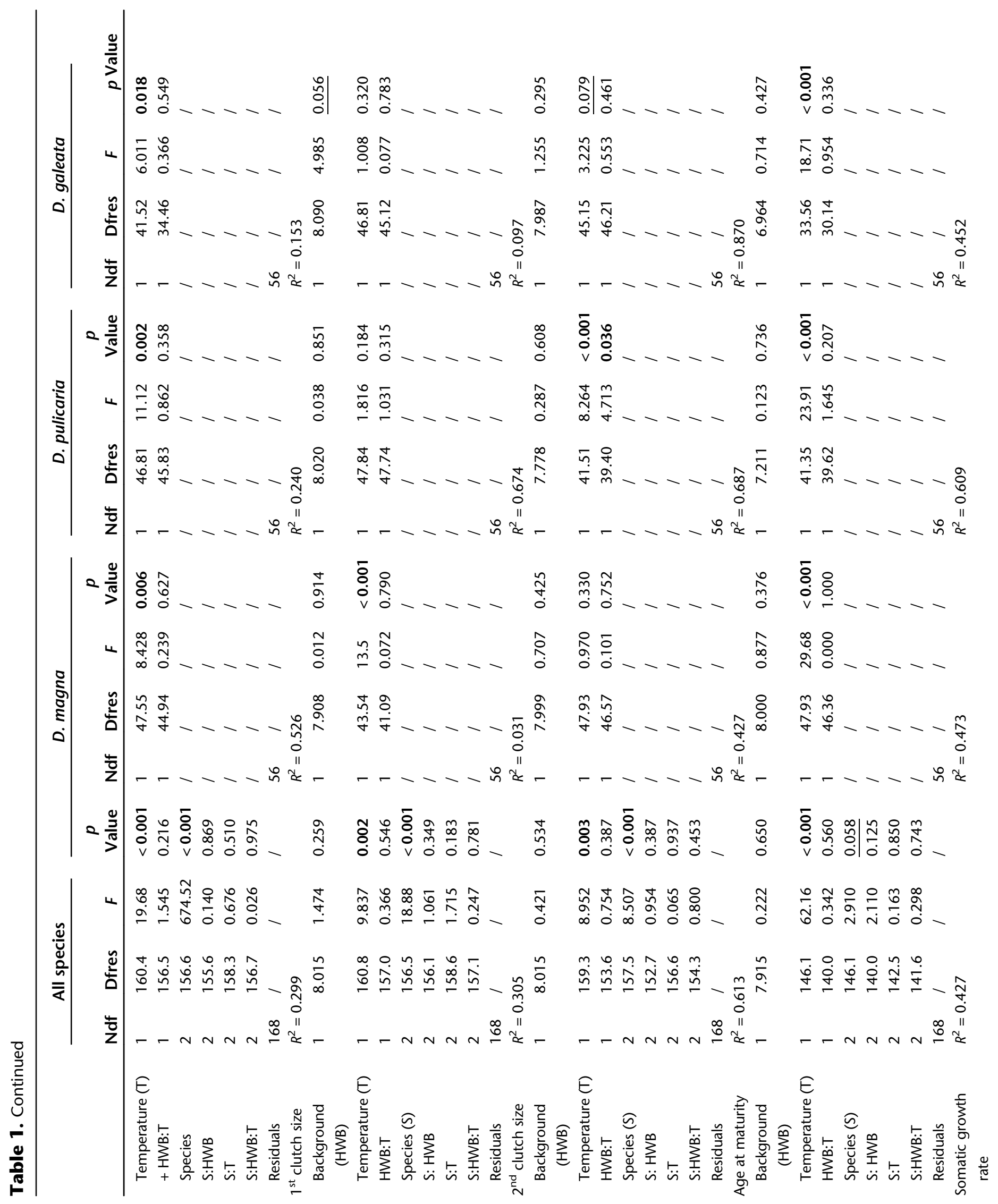




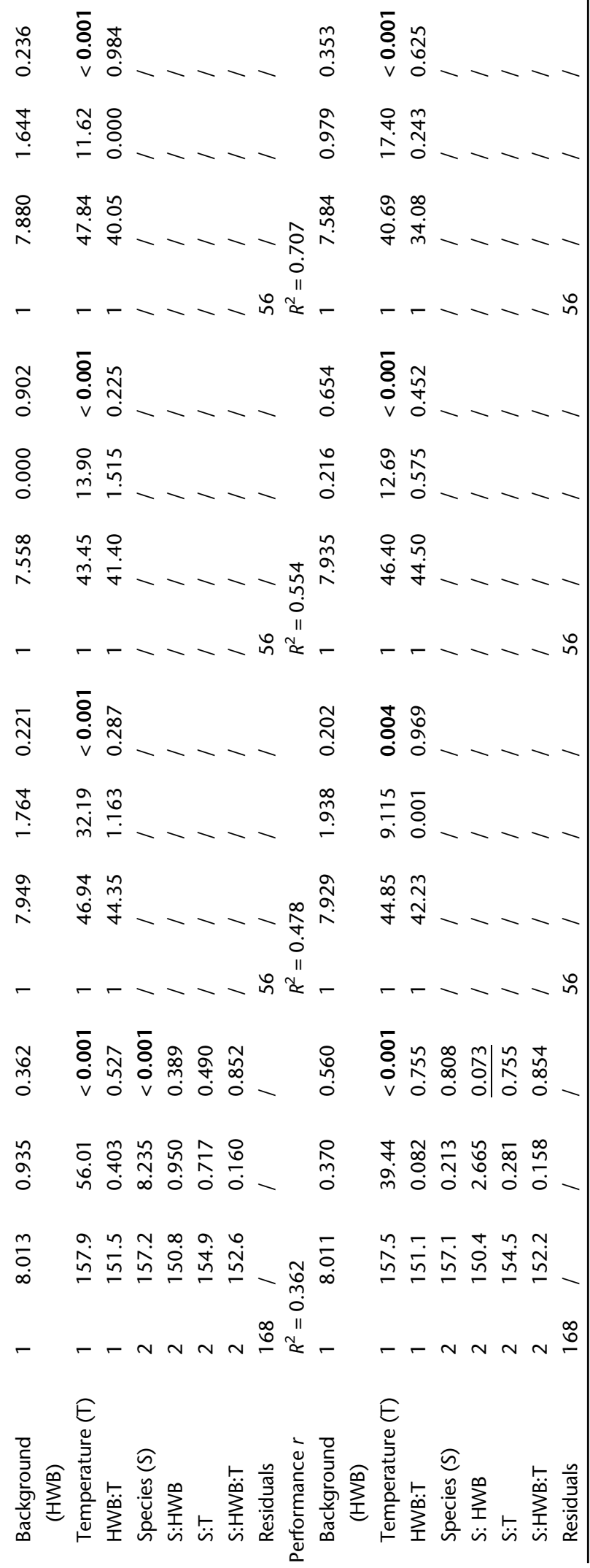

Moreover, because we only selected five clonal lineages per population, the likelihood that we captured the full range of clonal means in the population is low. Hence, our analysis likely underestimates the range of intraspecific variation, and is thus conservative in terms of estimating the degree of intraspecific compared to interspecific trait variation. As a final note, as our analysis is entirely based on adult individuals, we do not consider the contribution of ontogenetic effects to our estimate of intraspecific trait variation.

Last, to assess whether trait differences of species pairs converged or diverged upon exposure to a heat wave, we calculated the change between observed interspecific differences during the heat wave (exposed population at $26^{\circ} \mathrm{C}$; SP1 $1_{\text {Exposed,26 }}-\mathrm{SP} 2_{\text {Exposed,26) }}$ and the observed interspecific differences before warming (original population at $20^{\circ} \mathrm{C}$; $\mathrm{SP} 1_{\text {Original,20 }}-\mathrm{SP} 2_{\text {Original,20 }}$ ) for each species pair, as described previously. The index of divergence $\rho$ was calculated as the total difference of the absolute values of these interspecific differences for each species pair as follows:

$\rho=\operatorname{abs}\left(\mathrm{SP} 1_{\text {Exposed,26 }}-\mathrm{SP} 2_{\text {Exposed,26 }}\right)-\mathrm{abs}\left(\mathrm{SP} 1_{\text {Original,20 }}-\mathrm{SP} 2_{\text {Original,20 }}\right)$.

If this index is larger than 0 for a given trait and species pair, then the species have diverged in their trait response upon exposure to a heat wave. If this index is smaller than 0 for a given trait and species pair, it indicates that the two species are converging in trait values (i.e., become more similar) upon exposure to a heat wave. To determine the robustness of this index, we performed a bootstrap analysis, where we sampled with replacement the clonal trait means within populations per species and calculated the index of divergence for 1000 bootstrap samples to generate $95 \%$ confidence intervals. If $95 \%$ confidence intervals overlap with 0 , we cannot conclude a significant divergence or convergence occurred.

All visualizations were made using the package "ggplot2" (Wickham 2016). All statistical analyses were executed in RStudio Version 1.2.1335 and R Version 3.6.0 (R Core Team 2013).

\section{Results}

Effects of species, selection, and rearing temperature

The common garden data involving both the original nonexposed and the heat wave-exposed populations reveal highly significant differences among species for all traits combined as well as for five out of the seven traits when considered separately: $\mathrm{CT}_{\max }$, size at maturity, size of the $1^{\text {st }}$ and $2^{\text {nd }}$ clutches, and somatic growth rate (all $p<0.001$, df $=2$; Table 1). Age at maturity $(p=0.058, \mathrm{df}=2)$ and performance $r$ $(p=0.808, \mathrm{df}=2)$ do not differ significantly among species (Table 1). We find strongest differences between species for size at maturity (Fig. 2b). Trait differences are more pronounced between D. magna and the other two species, and 
less between D. pulicaria and D. galeata, with the difference between $D$. magna and $D$. galeata being significant for five traits (Table S1). For example, D. magna has an average $\mathrm{CT}_{\max }$ value around $41.06^{\circ} \mathrm{C}$, while the other two Daphnia species have a lower, but similar to each other average $\mathrm{CT}_{\max }$ value, $39.39^{\circ} \mathrm{C}$ for $D$. pulicaria and $39.06^{\circ} \mathrm{C}$ for $D$. galeata. Similarly, $D$. magna has a larger $1^{\text {st }}$ clutch size (6.71) than D. pulicaria (4.86) and D. galeata (4.06).

The overarching MANOVA also indicates a significant overall difference among rearing temperature and among species (both $p<0.001, \mathrm{df}=1$ and 2, respectively; Table 1). This pattern of interspecific differences and significant plasticity is corroborated for all (plasticity) or five out of the seven (interspecific differences) traits when they are analyzed separately. There is no significant overall effect of background (heat wave-exposed vs. original population, i.e., reflecting evolution) when traits are analyzed together or separately. There is, however, a significant interaction between species $\times$ background $\times$ temperature for CTmax $(p=0.004, \mathrm{df}=2)$ when analyzing the traits separately, indicating that species differ in their evolution of plasticity to the heat wave.

The MANOVAs on the three species separately confirm the highly significant effect of rearing temperature on trait values for each of the three species (Table 1). Both $D$. pulicaria and D. galeata show significant plasticity effects for $\mathrm{CT}_{\max }(p<0.001$, $\mathrm{df}=1$ ), with both species showing overall higher $\mathrm{CT}_{\max }$ values when the animals are cultured at a higher temperature (Fig. 2). There is no significant plasticity for $\mathrm{CT}_{\max }$ for D. magna. $D$. pulicaria also shows a significant background $\times$ temperature interaction for $\mathrm{CT}_{\max }$, reflecting evolution of plasticity toward a stronger response to warming in the exposed population vs. the original population.

All species exhibit phenotypic plasticity in size at maturity $(p<0.02, \mathrm{df}=1$; Table 1$)$. We have no evidence for evolution of plasticity, and overall plasticity responses are quite similar among species: in all three species there is a decline in size at
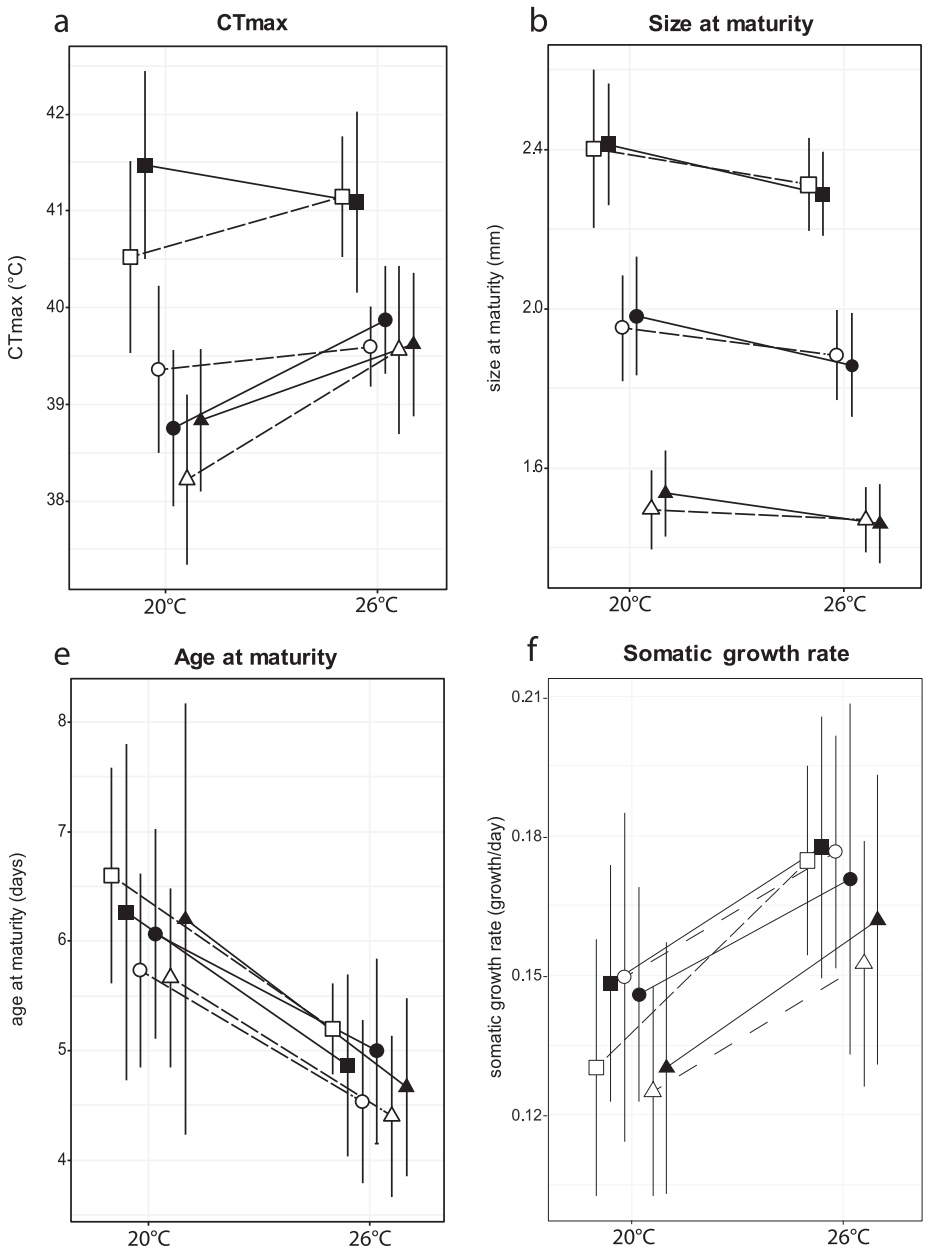
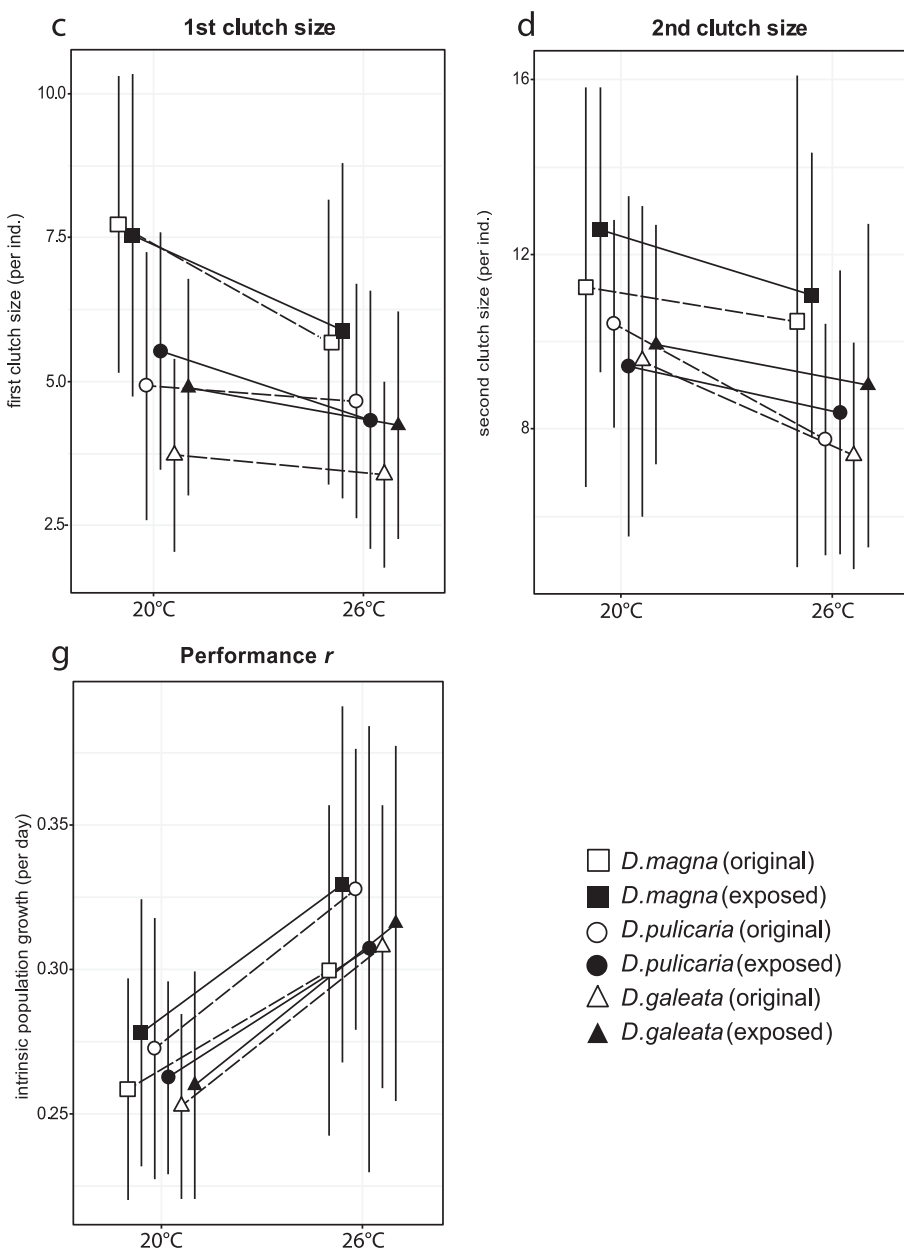

D.magna (original)

D.magna (exposed) D.pulicaria (original)

D.pulicaria(exposed)

$\triangle$ D.galeata (original)

A D.galeata (exposed)

Fig 2. Reaction norms for (a) heat tolerance $\left(\mathrm{CT}_{\max }\right)$, (b-f) five life-history traits and (g) performance $r$ for three Daphnia species (Daphnia magna square, Daphnia pulicaria circle, Daphnia galeata triangle) across two temperatures $\left(20^{\circ} \mathrm{C}\right.$ and $\left.26^{\circ} \mathrm{C}\right)$. Symbols represent species mean trait values for both the heat wave-exposed populations (isolated from mesocosm populations exposed to a heat wave; filled symbols) and the original population (hatched from the dormant egg bank of Langerodevijver; empty symbols). Error bars reflect \pm 1 standard deviation. 
maturity when reared at $26^{\circ} \mathrm{C}$ compared to $20^{\circ} \mathrm{C}$ (Fig. $2 \mathrm{~b}$ ). Only D. magna exhibits plasticity in $1^{\text {st }}$ clutch size $(p<0.001$, $\mathrm{df}=1$, Table 1 ), with smaller clutch sizes at the higher compared to the lower rearing temperature (Fig. 2c). In contrast to the response observed for the $1^{\text {st }}$ clutch size, we only find a significant effect of rearing temperature in $2^{\text {nd }}$ clutch size for $D$. pulicaria $(p<0.001, \mathrm{df}=1)$, with again a lower number of eggs at $26^{\circ} \mathrm{C}$ compared to $20^{\circ} \mathrm{C}$. For this species and trait, we also observe a significant temperature $\times$ background interaction effect ( $p=0.036, \mathrm{df}=1$, Table 1 ). This reflects that the

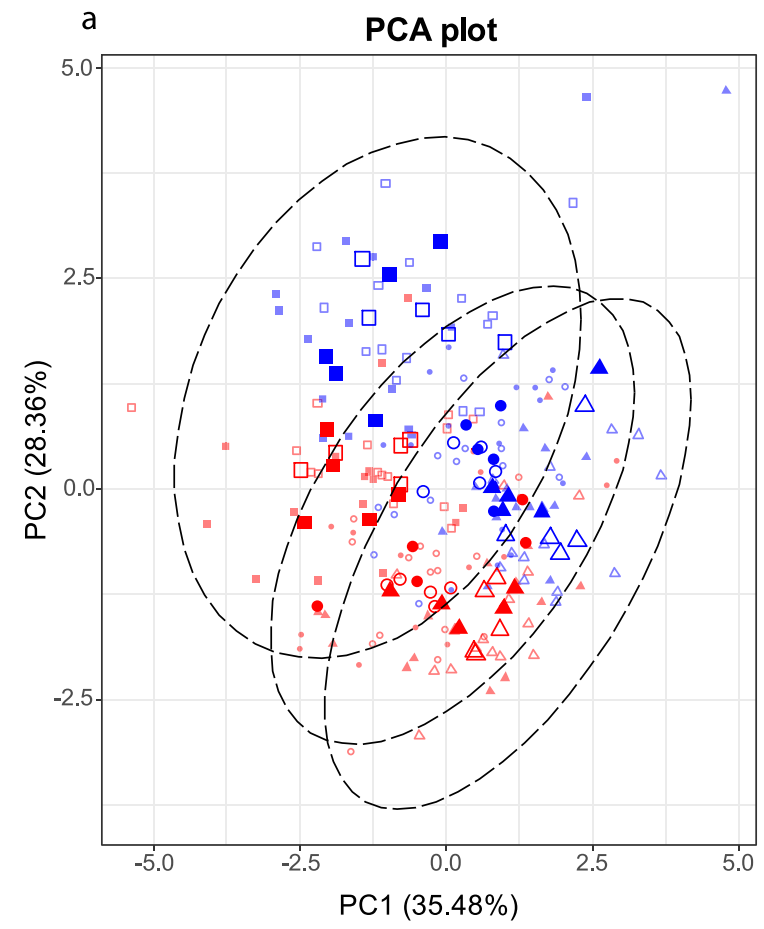

\section{Legend}

$\square$ D. magna (original)

- D. magna (exposed)

D D. pulicaria (original)

- D. pulicaria (exposed)

$\triangle$ D. galeata (original)

$\Delta$ D. galeata (exposed)
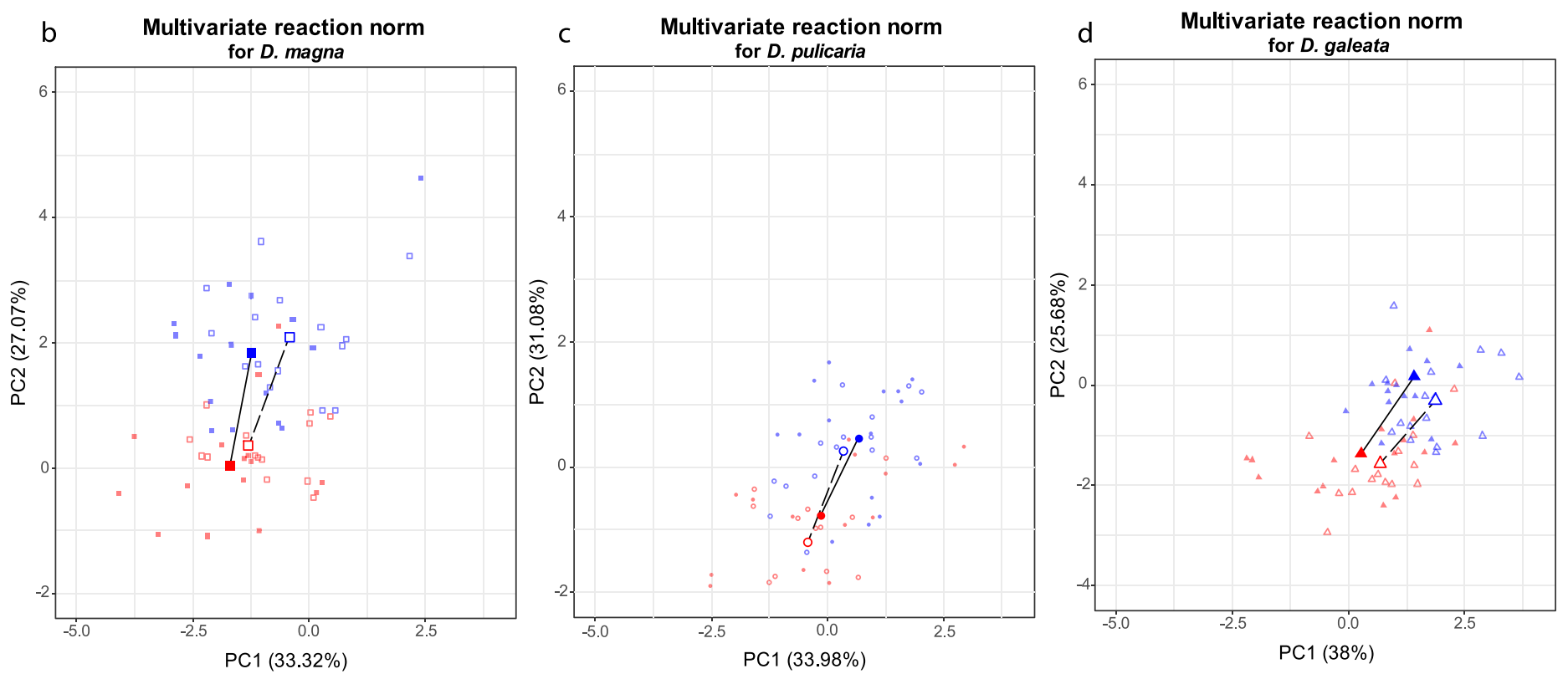

Fig 3. Visualization of multivariate trait space using a principle component analysis (PCA) reflecting the differences among three Daphnia species, selection backgrounds (heat wave-exposed vs. nonexposed original population) and culture temperatures (blue: $20^{\circ} \mathrm{C}$ and red: $26^{\circ} \mathrm{C}$ ) in multivariate space determined by their heat tolerance and life-history traits (a); and multivariate reaction norms in multivariate space for Daphnia magna (b), Daphnia pulicaria (c), and Daphnia galeata (d) using similar symbols and coloring. The larger symbols represent the population average, while the smaller symbols are the values for clonal lineages (averaged over replicates). 
decrease in clutch size at the higher temperature is lower in the heat wave-exposed population compared to the original population (Fig. 2d). All three species show significant plasticity $(p<0.001, \mathrm{df}=1$, Table 1$)$ for age at maturity, with an overall very similar decrease in time to maturity in the high temperature treatment. All three species exhibit plasticity for somatic growth rate and performance $r$ (all $p<0.005$, df $=1$, Table 1). Somatic growth rate and performance $r$ for all species are higher when animals are cultured at the higher compared to the lower temperature (Fig. 2f-g).

Overall, there is a significant plasticity response (i.e., main effect of temperature) in 16 out of 21 species $\times$ trait combinations, the only exceptions being $\mathrm{CT}_{\max }$ in $D$. magna, size of the $1^{\text {st }}$ clutch in both $D$. pulicaria and D. galeata, and size of the $2^{\text {nd }}$ clutch in D. magna and D. galeata (Table 1 ). The main effect of selection background is not significant for any of the traits in any of the species, reflecting that there was no significant evolution of mean trait values for any of the traits in the mesocosms during the heat wave. For $\mathrm{CT}_{\max }$ and size of the $2^{\text {nd }}$ clutch in D. pulicaria, however, we observed a significant background $\times$ temperature interaction.

To visualize the multivariate reaction norms, we performed a principal component analysis (Fig. 3). The $1^{\text {st }}$ axis (PC1) of the principal component analysis explains 35.48\% of the variation and correlates negatively with $\mathrm{CT}_{\max }$ $(-0.35)$, size of the $1^{\text {st }}(-0.41)$ and $2^{\text {nd }}$ clutches $(-0.31)$, somatic growth rate $(-0.42)$ and performance measure $r(-0.48)$, whereas the $2^{\text {nd }}$ axis (PC2) explains $28.36 \%$ of the a

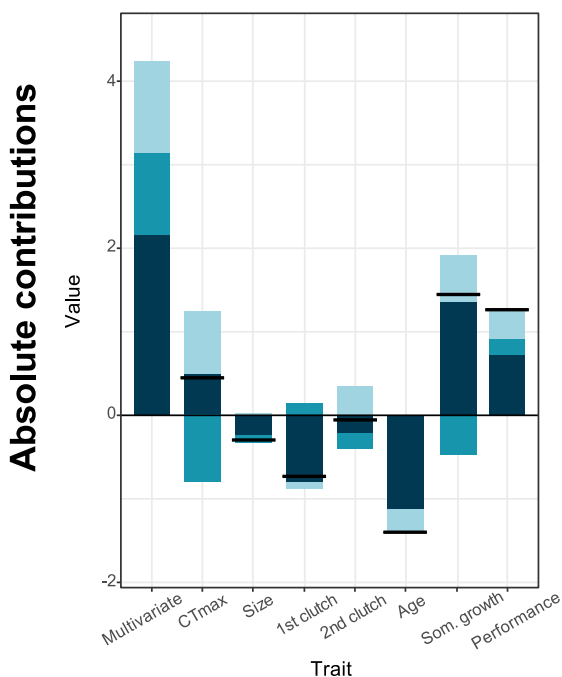

d

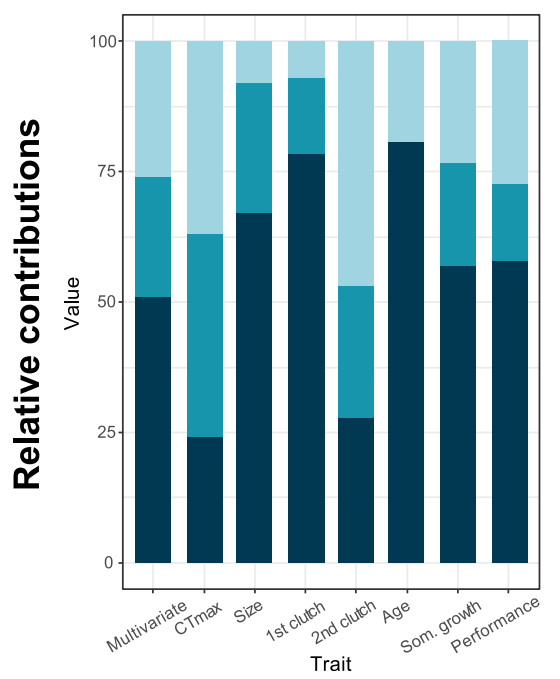

b

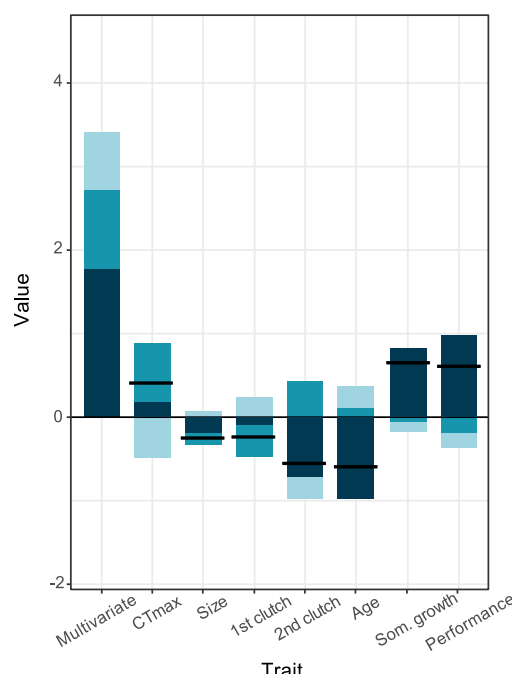

e

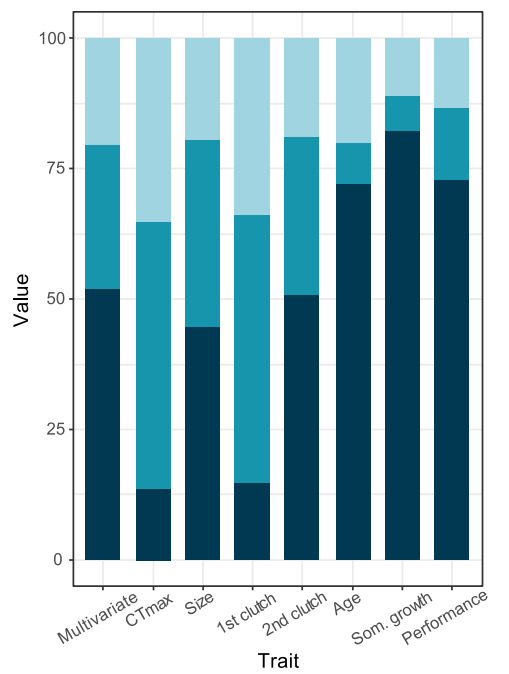

C

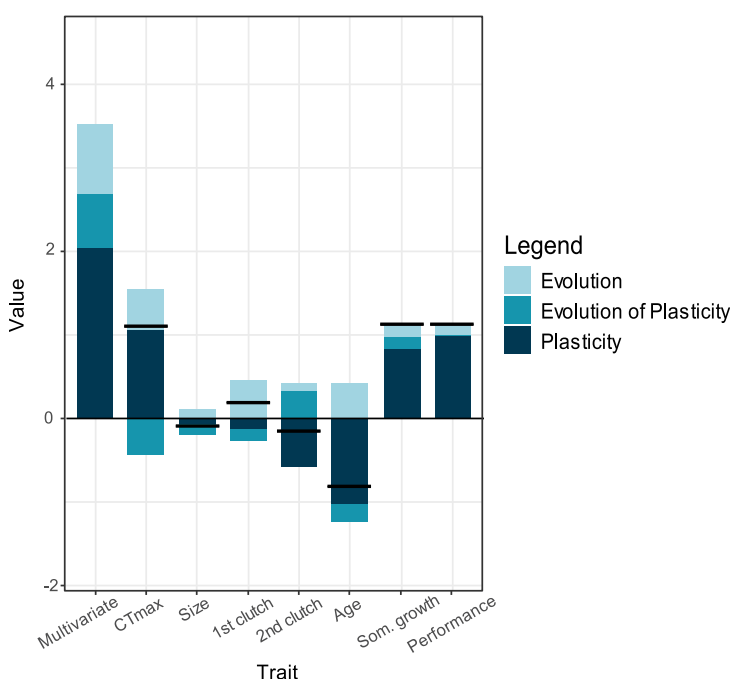

f

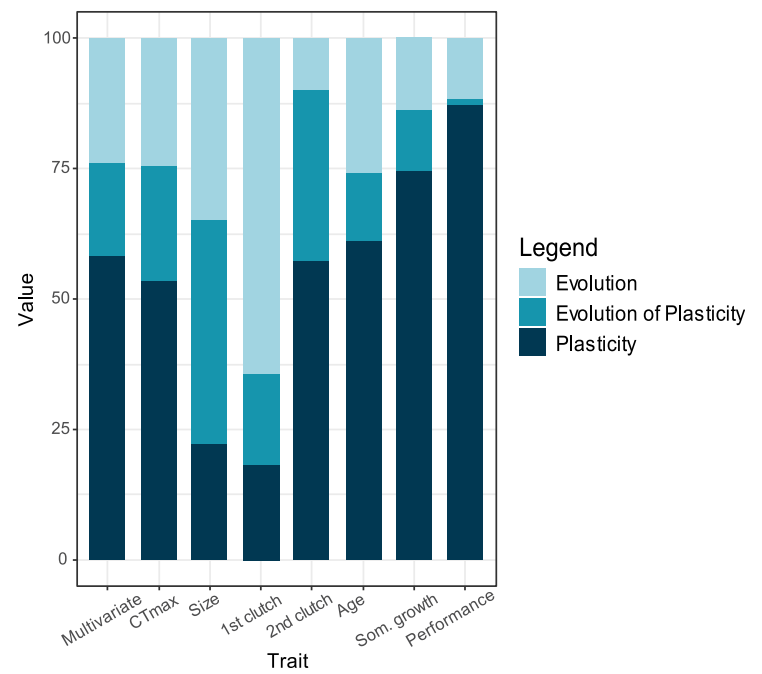

Fig 4. Absolute (a-c) and relative (d-e) contributions of ancestral phenotypic plasticity (dark blue), evolution of mean trait value (blue) and evolution of phenotypic plasticity (light blue) to total trait change (horizontal line in panels a-c) from the original population growing at $20^{\circ} \mathrm{C}$ to the heat waveexposed population growing at $26^{\circ} \mathrm{C}$ for the three study species. 
variation and correlates positively with size (0.44) and age at maturity (0.61; Table S2). Also in multivariate trait space, we observe clear differences among species and the effect of temperature on trait values (Fig. 3a). The scores of $D$. pulicaria are intermediate to those of $D$. galeata and D. magna. The response to temperature is distinguishable along PC2, and shows a similar trend across species (Fig. 3a). The multivariate reaction norms of all species and of both the exposed and original populations of D. magna, D. pulicaria, and $D$. galeata all tend to run parallel, which indicates that there is a comparable response to an increase in temperature across species and across populations that were and were not exposed to a heat wave in a mesocosm experiment (Fig. 3b-d).
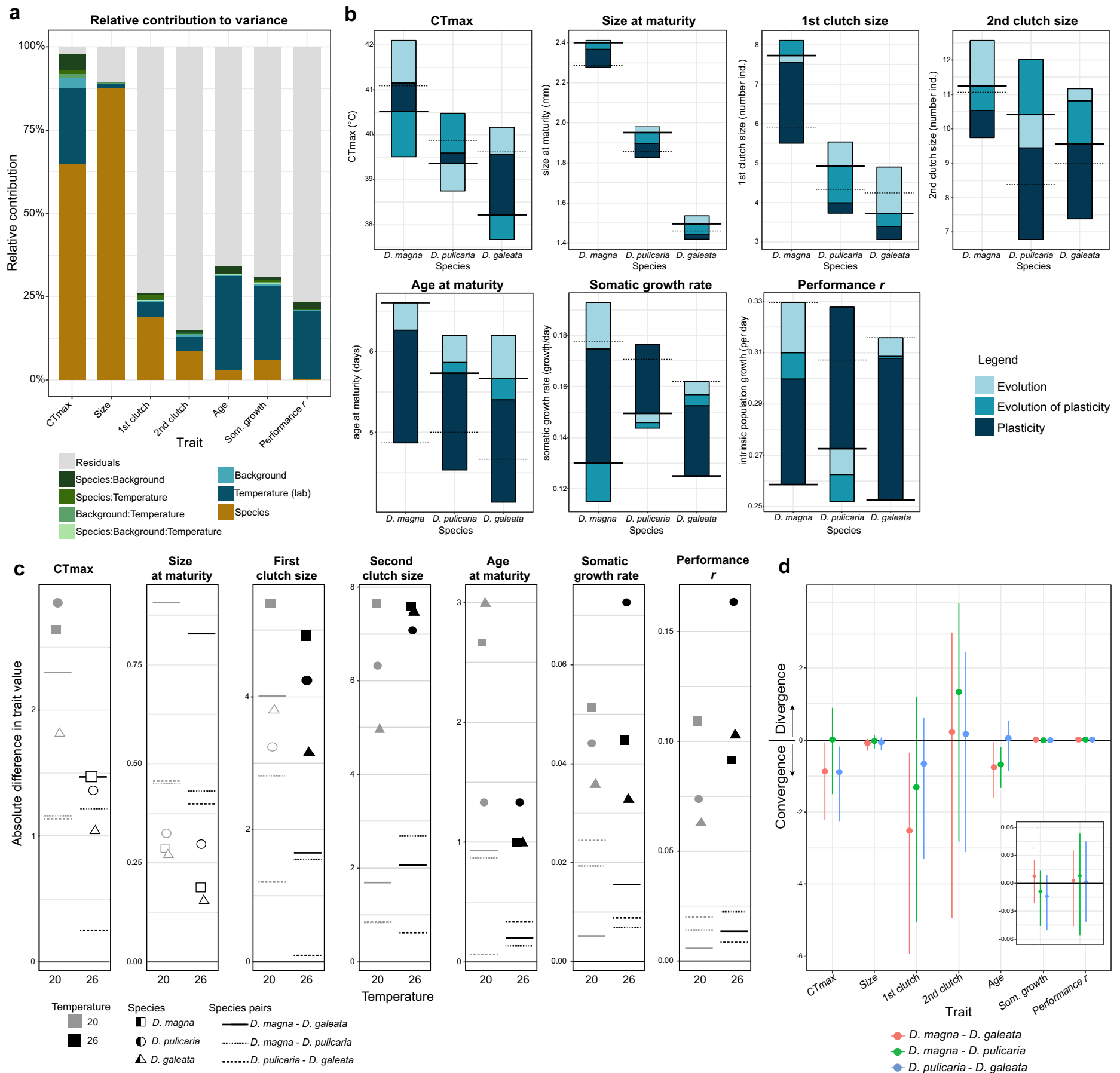

Fig 5. Legend on next page. 


\section{Contribution of plasticity and evolution to trait change}

Absolute and relative contributions to the total trait change from the original population reared at average summer temperatures $\left(20^{\circ} \mathrm{C}\right)$ to the heat wave-exposed population reared at the higher thermal regime $\left(26^{\circ} \mathrm{C}\right)$ are shown in Fig. 4 . The total trait change observed for each trait is similar for the three species, but how the different eco-evo components contribute to it differs among traits and species (Fig. 4). For example, for heat tolerance, we found an increase in total heat tolerance from the original nonexposed populations (at $20^{\circ} \mathrm{C}$ ) to the exposed populations (at $26^{\circ} \mathrm{C}$ ) for the three species, but the contribution of evolution of plasticity was for $D$. pulicaria in the same direction as that of total trait change, while for $D$. magna and $D$. galeata evolution of plasticity resulted in a trait change that was in the opposite direction of the net total change. Ancestral phenotypic plasticity, however, was for all traits in the same direction across species.

Overall, the observed net changes in trait values (Fig. 4) are in line with the idea that the plastic responses are adaptive to warming: the net increase in heat tolerance, reduction in age at maturity, increase in somatic growth rate and increase in performance $r$ all suggest that the species increase their performance at the high temperature $\left(\mathrm{CT}_{\max }\right)$ or that they show a higher fitness at the high temperature. There is a small net decrease in size at maturity in the three species. For clutch size, the pattern is more variable, with overall positive net effects on the size of the $1^{\text {st }}$ clutch and negative net effects on the $2^{\text {nd }}$ clutch.

The plots on the relative contribution of plasticity, evolution of mean trait value and evolution of plasticity to total trait change show that phenotypic plasticity is often very important (Fig. 4). In 14 out of the 21 species $\times$ trait analyses, phenotypic plasticity in the original population accounted for more than $50 \%$ of total trait change. For heat tolerance $\left(\mathrm{CT}_{\max }\right)$, however, evolution accounted for approximately $50 \%$ of total trait change in D. galeata and for more than $75 \%$ of total trait change in $D$. magna and D. pulicaria. In each of the three species, evolution of mean trait value and evolution of plasticity often had a similar contribution to net change in trait values, and in most cases they were in opposite direction of each other.

\section{Scaling intraspecific to interspecific differences}

The among-species differences for size at maturity are very large compared to the capacity of the different species to respond through phenotypic plasticity or evolutionary trait change (Fig. 5a-c; Table S3). Less pronounced, but still larger contribution of interspecific differences was also found for $\mathrm{CT}_{\max }$ and $1^{\text {st }}$ clutch size (Fig. $5 \mathrm{a}-\mathrm{c}$ ). For both of these traits we found a significant convergence between D. magna and D. galeata upon warming, indicating that $\mathrm{CT}_{\max }$ and $1^{\text {st }}$ clutch size values became more similar in the heat wave-exposed population compared to the original nonexposed population of these species (Fig. 5d). For $\mathrm{CT}_{\max }$, this was also observed between $D$. pulicaria and D. galeata. For $2^{\text {nd }}$ clutch size, age at maturity, somatic growth rate and performance $r$ the observed net change in trait values achieved by changes in trait values within species is relatively large compared to the interspecific differences observed for these traits (Fig. 5a,c; Table S3). The relative contribution of intraspecific trait change compared to interspecific differences is largest for age at maturity and performance $r$ (Fig. 5c). Exposure to a heat wave results in trait convergence between $D$. magna and D. galeata and D. magna and $D$. pulicaria for age at maturity (Fig. 5d), even though trait differences among these species were already small at $20^{\circ} \mathrm{C}$.

\section{Discussion}

Climate warming increases the likelihood of extreme events such as heat waves, and it is likely that these will become major selection factors determining the success of species and the functioning of ecosystems (Hendry et al. 2017; Lancaster et al. 2017). Understanding how species and communities

FIG 5. (a) Relative contributions of inter- and intraspecific trait variance for each trait using regression sum of squares obtained from ANOVA. Interspecific variation is given by the main effect of species (brown), and intraspecific variation is represented by the main effects of selection background (i.e., exposure to heat wave), rearing laboratory temperatures (different hues of blue), and all interactive effects (different hues of green). Unexplained variation (i.e., residuals) was also plotted (gray). (b) Absolute contribution of ancestral phenotypic plasticity (dark blue), evolution of mean trait value (light blue) and evolution of plasticity (blue) to total trait change per species, positioned on a common trait axis so that the amplitude of within-species trait change can be compared to the differentiation in trait values among species. The solid (resp. dashed) line indicates the trait value of the original population reared at $20^{\circ} \mathrm{C}$ (resp. of the heat wave-exposed population reared at $26^{\circ} \mathrm{C}$ ). The figure illustrates that the different components can have different directions, so that large individual components can sometimes compensate for each other resulting in a modest total trait change. (c) Visualization of the absolute intra- and interspecific trait differences. Intraspecific trait variation (symbols: Daphnia magna = squares; Daphnia pulicaria = circles; Daphnia galeat $=$ triangles) is calculated as the maximum difference between the most extreme clonal lineages trait values (gray colors: original population at $20^{\circ} \mathrm{C}$; black colors: exposed population at $26^{\circ} \mathrm{C}$ ). Interspecific differences for each species pair (horizontal lines: solid line for $D$. magna and $D$. galeata; dotted line for $D$. magna and $D$. pulicaria, and dashed line for $D$. pulicaria and $D$. galeata) is calculated as the difference between species trait means (gray colors: species pairs from original population at $20^{\circ} \mathrm{C}$; black colors: species pairs from exposed population at $26^{\circ} \mathrm{C}$ ). The position of the symbols and the lines visualize whether intra- or interspecific variation can contribute more to the change in trait values for the set of three Daphnia species studied. the symbols are filled if the intraspecific variation is larger than the interspecific variation and are empty when the opposite is true. (d) the change between the interspecific differences before (original populations cultured at $20^{\circ} \mathrm{C}$ ) and after (evolved populations cultured at $26^{\circ} \mathrm{C}$ ) heat wave exposure, illustrating whether the species converged $(<0)$ or diverged $(>0)$ in trait value upon exposure to a heat wave. error bars reflect $95 \%$ quantiles to visualize the degree to which there is overlap with 0 , reflecting no significant divergence or convergence. This was calculated for each species pair (red: D.magna and D. galeata; green: D. magna and D. pulicaria and blue: D. pulicaria and D. galeata). The zoomed insert shows the error bars for somatic growth rate and performance $r$. 
respond to heat waves will therefore be key to better predict ecological responses to global change (Urban et al. 2016). However, many studies focus on understanding responses of a single species, while it is important to take a broader perspective and link population to community responses. Our study is a first step toward including a multiple species perspective (De Meester et al. 2019) and integrating trait responses at the species and community level (Govaert et al. 2016). Key to this is determining the relative importance of intraspecific trait variation vs. trait differentiation among species (Brans et al. 2017a; Des Roches et al. 2018).

In this study, we took advantage of a naturally occurring heat wave to quantify how populations of three co-occurring congeneric species-three Daphnia species isolated from the same pond-respond to a heat wave in terms of their trait values for both heat tolerance and multiple life-history traits. We quantified (1) the extent to which each species showed an evolutionary trait change to a heat wave, (2) the relative importance of phenotypic plasticity, evolution of mean trait values, and evolution of plasticity to total trait change within each of the three species, and (3) how the change in intraspecific trait variation in response to the heat wave alters the amount of interspecific trait differentiation. Our results show strong changes in trait values across the three species for most of the traits studied. Most of these changes are in line with expectations from adaptive responses to warming. We found that phenotypic plasticity to a higher rearing temperature $\left(20^{\circ} \mathrm{C}\right.$ vs. $\left.26^{\circ} \mathrm{C}\right)$ is a major component of trait change for at least five out of the seven traits for each species. While evidence for microevolutionary responses is more limited, we did observe a significant effect of evolution of plasticity for some traits $\times$ species combinations. In terms of effect size, the contribution of evolutionary trait change to total trait change under a heat wave scenario should for some traits not be underestimated, and was often higher than $25 \%$ (e.g., heat tolerance). In addition, for several traits (i.e., $2^{\text {nd }}$ clutch size, age at maturity, somatic growth rate, and performance $r$ ) the amount of intraspecific trait variation is quite large compared to the among-species differences in trait values, suggesting that intraspecific trait variation might contribute significantly to community trait responses to extreme events. Size at maturity is an exception, as for this trait the intraspecific variation contributes little to the degree to which species differ in trait values. In the following paragraphs we discuss these results in more detail and point to methodological considerations.

We observed significant changes in trait values in response to the heat wave for all studied traits. While evidence for evolutionary trait shifts was mixed, all traits showed phenotypic plasticity in response to a temperature increase for at least one of the three species. For all traits except heat tolerance, the phenotypic plasticity response did not differ across the three species. The direction of trait change mediated by phenotypic plasticity generally was in line with expectations for adaptive responses to warming. For instance, age at maturity was reduced at higher temperatures, which reflects the dependence of developmental time on temperature (Ranta et al. 1993; Chopelet et al. 2008). Size at maturity was slightly reduced at higher temperature, which is in line with the temperature size rule (Atkinson 1994) and earlier results on Daphnia (Ranta et al. 1993; Chopelet et al. 2008). Given the shorter development time and the fact that size was only slightly reduced, somatic growth rate was higher at $26^{\circ} \mathrm{C}$ compared to $20^{\circ} \mathrm{C}$. This is in line with observations reported in previous studies (Mitchell and Lampert 2000; Herrmann et al. 2017). While the size of the $1^{\text {st }}$ and $2^{\text {nd }}$ clutch tended to decrease, the strong reduction in age at maturity resulted in a strong increase in intrinsic population growth rate (Mitchell and Lampert 2000). In line with an interpretation as an adaptive response to warming, heat tolerance overall showed an increase when animals were cultured at higher temperatures. This response was the least strong for Daphnia pulicaria.

While phenotypic plasticity contributed more to total trait change upon warming than evolutionary trait change for most traits, we observed significant evolutionary trait change for heat tolerance and size of $2^{\text {nd }}$ clutch in D. pulicaria. This needs to be viewed in the context that our selection experiment only lasted 6 weeks. Our results thus show that exposure to naturally occurring heat waves can induce significant evolutionary responses in natural Daphnia populations, even within a few weeks. This is in line with the results of other mesocosm studies that focused on other selection factors, such as exposure to an insecticide (Jansen et al. 2015). It highlights that the evolutionary response to warming that we observed in a mesocosm experiment that lasted for 2 years (Geerts et al. 2015) might have (in part) occurred within one growing season. We also stress that we inoculated a limited set of clones directly hatched from the natural dormant egg bank of a single population, thus representing a subset of the genetic diversity that one might expect at the start of the growing season in that study population. The evolutionary response that we observe reflects existing standing genetic variation and indicates that natural Daphnia populations harbor sufficient genetic variation to show evolutionary responses when exposed to heat waves. Heat waves can, through the evolutionary responses that they can induce, have a legacy effect on the characteristics and dynamics of populations in the remainder of the growing season. While we do show that natural Daphnia populations can evolve when exposed to a heat wave, it should also be stressed that there are clear limits to its effectiveness. This is reflected by our observation that nearly all D. pulicaria and D. galeata populations went extinct in the heated treatment, in which we imposed another $4^{\circ} \mathrm{C}$ temperature increase on top of the heat wave. The fact that most D. magna populations survived in the heated mesocosms is in line with our observation that $\mathrm{CT}_{\max }$ of $D$. magna is higher than that of D. pulicaria and D. galeata.

Our results show an interesting contrast when focusing on significance vs. effect size. In our MANOVA and ANOVA 
analyses, the effect of phenotypic plasticity is significant for most traits $\times$ species combinations, whereas evolutionary trait change is only significant in two out of 21 trait $\times$ species combinations. This suggests that the scope for evolutionary responses to impact trait change is very limited in this combination of selection agent, time frame, environmental conditions, measured traits and species. Yet, our effect size analysis provides a somewhat more nuanced picture, as it focuses more on the biological importance of these processes rather than the statistical significance (Nakagawa and Cuthill 2007). While phenotypic plasticity remains the most important driver of total trait change upon exposure to a heat wave, evolutionary trait change represents more than $25 \%$ of total trait change under a heat wave scenario in 17 out of 21 trait $\times$ species combinations. In five trait $\times$ species combinations, evolutionary trait change contributes even more than $75 \%$ to total trait change.

In 14 out of the 21 trait $\times$ species combinations, the net impact of evolutionary trait change is reduced due to opposite direction of evolution of mean trait value and evolution of phenotypic plasticity. This is in accordance with previous findings in other studies (Ghalambor et al. 2015; Stoks et al. 2016). Plastic responses can develop quickly in response to environmental variation, while evolutionary responses take more time to develop. As environments fluctuate, it might be that the short-term responses to abiotic changes through phenotypic plasticity differ from the long-term response mediated by evolution of mean trait value and evolution of plasticity. The relationship between phenotypic plasticity and evolution of trait values is heavily debated (Lande 2009): while adaptive plasticity can slow down genetic adaptation by changing the distribution of phenotypes on which natural selection can act (Fox et al. 2019), other studies have shown that plasticity can facilitate adaptation via genetic accommodation (Braendle and Flatt 2006; Lande 2009). The fact that evolution of mean trait and evolution of plasticity differ in direction might result from trade-offs (Ghalambor et al. 2015). In the case of body size, for instance, phenotypic plasticity results in smaller animals upon warming. If warming also drives evolution towards smaller body sizes, then it might be that there is a concurrent selection to reduce plasticity because a too small body size has important fitness costs. Alternatively, if plasticity facilitates trait evolution by genetic assimilation, the resulting pattern is also that evolution is associated with reduced plasticity (Braendle and Flatt 2006). In an earlier study in which the relative importance of phenotypic plasticity and evolutionary trait change was quantified in a resurrection ecology analysis focusing on the response of a D. magna population to changes in fish predation pressure, the amplitude and direction of phenotypic plasticity, evolution of mean trait value and evolution of plasticity similarly differed strongly across traits (Stoks et al. 2016).

Our study is unique to the extent that we quantified phenotypic plasticity and evolutionary trait responses in seven traits (heat tolerance, five life-history traits and performance $r$ ) in three co-occurring species of the same genus (Daphnia) that are derived from a single pond. Such comparative studies involving multiple species of the same guild are not only important in revealing the generalities or peculiarities of responses observed in specific species, but in addition are crucial in helping us understand how intraspecific trait variation can translate into altered interspecific interactions. An important benchmark in this perspective is the relative amplitude of among-species differences and within-species trait variation. It is striking that especially size at maturity seems to show an almost fixed difference among the three co-occurring Daphnia species, whereas the interspecific differences in other traits is much reduced when scaled to intraspecific trait variation. Daphnia species are known to strongly differ in body size, which has been linked to both predator avoidance as well as competitive strength and capacity for top-down control of algae (Brooks and Dodson 1965; Goulden et al. 1982; Miner et al. 2012). Our results suggest that indeed body size might be the key trait structuring differences among Daphnia species, which is in line with the observation that body size contributes in important ways to explain local community structure (Gianuca et al. 2016a). While our results also confirm that these differences are associated with variation in other traits (cf. a slightly delayed age at maturity, larger clutch sizes and a higher somatic growth rate in D. magna compared to the other two species), the among-species variation in these traits is less pronounced than for size at maturity, and the relative importance of intraspecific trait variation is higher for these traits than for size at maturity.

While we studied phenotypic and evolutionary responses in three co-occurring Daphnia species, our design only still captures part of a true multispecies perspective (De Meester et al. 2019). This is because our study quantifies the response of all three species to a heat wave while they were grown in monoculture, and thus does not take the impact of species interactions on the evolutionary response into account (Barraclough 2015). Under natural conditions, the populations of the three species studied here co-occur in the same pond, and the direct and indirect interactions among the species might potentially strongly influence the evolutionary responses of each of the three species to heat waves. Ideally, comparative analyses are done in experiments in which multiple species are exposed to a selection pressure while either in monoculture or embedded in their natural community (De Meester et al. 2019).

The size temperature rule predicts a size reduction at higher temperatures because smaller animals can cope better with heat stress (Atkinson 1994). It has indeed been observed that Daphnia become smaller when grown at higher temperatures (Chopelet et al. 2008; Henning-Lucass et al. 2016), and earlier work documented that D. magna populations exposed to higher temperatures (Geerts et al. 2015) or isolated from urban compared to rural habitats, and thus exposed to the urban heat island effect, are both smaller and have a higher heat 
tolerance (Brans et al. 2017b). Yet, the association between body size and heat tolerance is in our study lost when we compare the responses of different species, as we observe that D. magna has a higher heat tolerance than the smaller D. pulicaria and D. galeata. This illustrates that heat tolerance is not solely a function of body size, but also reflects physiological and biochemical adaptations such as the presence of heat-shock proteins or hemoglobin variants with a strong affinity for oxygen (Zeis et al. 2013; Brans et al. 2017b).

Our approach has several strengths. First, we worked with a representative sample of standing genetic variation present in natural populations of three different species co-occurring in a single habitat. Second, we kept all clones for several generations under standardized common garden conditions so that the observed differences among species and clones are purged from interference from maternal effects. Third, we did not only assess responses to a natural occurring heat wave but also exposed our experimental animals to two different temperatures during the common garden thermal tolerance and life table experiment so that we could quantify the relative importance of phenotypic plasticity and evolutionary responses. The major limitation of our study lies in the fact that we compared the heat wave-exposed populations to the original population directly hatched from the dormant egg bank. While this comparison is fully warranted in terms of assessing evolutionary responses, we have to be cautious to attribute these evolutionary responses solely to the heat wave. Given that we capitalized on the occurrence of a pronounced heat wave, we have no control population that was exposed to the mesocosm conditions but did not experience the heat wave. Originally our design did contain a warming and a control condition, but due to the heat wave we lost the warming treatment because nearly all populations of two of the three species went extinct in this $+4^{\circ} \mathrm{C}$ warming treatment. We then took the opportunity to compare the populations that were exposed to the heat wave to the original population, but we need to acknowledge that our observed responses to the heat wave might be confounded with responses that reflect adaptation to the conditions that prevailed in the mesocosms. Yet, while it is more difficult to make a direct link to heat wave exposure for the observed evolutionary responses in lifehistory traits (heat tolerance and size of the $2^{\text {nd }}$ clutch in D. pulicaria), it is more straightforward to attribute the evolutionary response in heat tolerance to the heat wave rather than to exposure to mesocosm conditions.

Our study shows how phenotypic plasticity and evolutionary trait change contribute to trait change in heat tolerance and five life-history traits and performance $r$, across three species in a mesocosm experiment during which the populations were exposed to one of the pronounced heat waves that characterized the summer of 2018 in Western Europe. Our results provide evidence for substantial evolution of plasticity, even though for most traits and species phenotypic plasticity dominated the observed trait change. The relative amplitude of intraspecific variation compared to interspecific trait differentiation differed strikingly among traits, with size at maturity being the trait that was most consistently different among species. Overall, it seems that the three test species are more likely to converge rather than to diverge in trait values after exposure to warming, as seen in Fig. $5 \mathrm{~d}$. This could have large implications for community level responses to warming. Trait convergence might both increase niche overlap as well as equalize fitness differences among species (Chesson 2000). In our study, however, body size, which is a strong determinant of competitive ability in Daphnia, was the trait that remained most consistently different among species, in line with body size being a master trait structuring zooplankton communities (Goulden et al. 1982; Gianuca et al. 2016b). Changes in interspecific differences upon exposure to a heat wave may affect direct and indirect species interactions, the effects of which can ripple through to other trophic levels (Urban et al. 2016). Interspecific trait convergence may also affect ecosystem functioning, among others by narrowing trait distributions that provide the raw material for ecosystems to respond to perturbations. The robustness of ecosystems depends on the diversity in trait values at the community level and on functional redundancy. Niche complementarity can also contribute to an increased performance of diverse compared to less diverse communities (Cook-Patton et al. 2011).

An increased effort in developing a multiple species perspective and integrating population and community responses as well as evolutionary and nonevolutionary responses will be important to make reliable predictions of responses to climate change (Urban et al. 2016). Studies such as the present one are only a first step, as a true multispecies perspective also will require studies that involve the analysis of eco-evolutionary dynamics being influenced by and influencing community composition (De Meester et al. 2019).

\section{References}

Altermatt, F., V. I. Pajunen, and D. Ebert. 2008. Climate change affects colonization dynamics in a metacommunity of three Daphnia species. Glob. Chang. Biol. 14: 1209-1220. doi:10.1111/j.1365-2486.2008.01588.x

Atkinson, D. 1994. Temperature and organism size-A biological law for ectotherms? Adv. Ecol. Res. 25: 1-58.

Barraclough, T. G. 2015. How do species interactions affect evolutionary dynamics across whole communities ? Annu. Rev. Ecol. Evol. Syst. 46: 25-48. doi:10.1146/annurevecolsys-112414-054030

Bottrell, H. H., and others. 1976. A review of some problems in zooplankton production studies. Nor. J. Zool. 24: 419-456.

Braendle, C., and T. Flatt. 2006. A role for genetic accommodation in evolution? Bioessays 28: 868-873. doi:10.1002/bies. 20456 
Brans, K. I., L. Govaert, J. M. T. Engelen, A. T. Gianuca, C. Souffreau, and L. De Meester. 2017a. Eco-evolutionary dynamics in urbanized landscapes: Evolution, species sorting and the change in zooplankton body size along urbanisation gradients. Philos. Trans. R. Soc. B. 372: 1-11. doi:10.1098/rstb.2016.0030

Brans, K. I., M. Jansen, J. Vanoverbeke, N. Tüzün, R. Stoks, and L. De Meester. 2017b. The heat is on: Genetic adaptation to urbanization mediated by thermal tolerance and body size. Glob. Chang. Biol. 23: 5218-5227. doi:10.1111/ gcb. 13784

Brooks, J. L., and S. I. Dodson. 1965. Predation, body size and composition of plankton. Science 150: 28-35. doi:10.1126/ science.150.3692.28

Carvalho, G. R. 1987. The clonal ecology of Daphnia magna (Crustacea: Cladocera): II. Thermal differentiation among seasonal clones. J. Anim. Ecol. 56: 469-478.

Chesson, P. 2000. Mechanisms of maintenance of species diversity. Annu. Rev. Ecol. Syst. 31: 343-366. doi:10.1146/ annurev.ecolsys.31.1.343

Chopelet, J., P. U. Blier, and F. Dufresne. 2008. Plasticity of growth rate and metabolism in Daphnia magna populations from different thermal habitats. J. Exp. Zool. 309A: 553-562. doi:10.1002/jez.488

Ciais, P., and others. 2005. Europe-wide reduction in primary productivity caused by the heat and drought in 2003. Nature 437: 529-533. doi:10.1038/nature03972

Cook-Patton, S. C., S. H. McArt, A. L. Parachnowitsch, J. S. Thaler, and A. A. Agrawal. 2011. A direct comparison of the consequences of plant genotypic and species diversity on communities and ecosystem function. Ecology 92: 915-923. doi:10.1890/10-0999.1

Daufresne, M., K. Lengfellner, and U. Sommer. 2009. Global warming benefits the small in aquatic ecosystems. Proc. Natl. Acad. Sci. 106: 12788-12793. doi:10.1073/pnas. 0902080106

De Meester, L., W. Van Doorslaer, A. N. Geerts, L. Orsini, and R. Stoks. 2011. Thermal genetic adaptation in the water flea Daphnia and its impact: An evolving metacommunity approach. Integr. Comp. Biol. 51: 703-718. doi:10.1093/ icb/icr027

De Meester, L., and others. 2019. Analysing eco-evolutionary dynamics-The challenging complexity of the real world. Funct. Ecol. 33: 43-59. doi:10.1111/1365-2435.13261

Des Roches, S., D. M. Post, N. E. Turley, J. K. Bailey, A. P. Hendry, M. T. Kinnison, J. A. Schweitzer, and E. P. Palkovacs. 2018. The ecological importance of intraspecific variation. Nat. Ecol. Evol. 2: 57-64. doi:10.1038/s41559017-0402-5

DeWitt, T. J., A. Sih, and D. Sloan Wilson. 1998. Costs and limits of phenotypic plasticity. Trends Ecol. Evol. 13: 77-81.

Ellner, S. P., M. A. Geber, and N. G. Hairston Jr. 2011. Does rapid evolution matter? Measuring the rate of contemporary evolution and its impacts on ecological dynamics. Ecol. Lett. 14: 603-614. doi:10.1111/j.1461-0248.2011.01616.X

Fox, J., and S. Weisberg. 2019. An R companion to Applied Regression. Thousend Oaks, CA. 3, SAGE Publications, Inc, Sage.

Fox, R. J., J. M. Donelson, C. Schunter, T. Ravasi, and J. D. Gaitán-Espitia. 2019. Beyond buying time: The role of plasticity in phenotypic adaptation to rapid environmental change. Phil. Trans. R. Soc. B 374: 1-9. doi:10.1098/rstb. 2018.0174

Geerts, A. N., and others. 2015. Rapid evolution of thermal tolerance in the water flea Daphnia. Nat. Clim. Chang. 5: 665-668. doi:10.1038/nclimate2628

Ghalambor, C. K., K. L. Hoke, E. W. Ruell, E. K. Fischer, D. N. Reznick, and K. A. Hughes. 2015. Non-adaptive plasticity potentiates rapid adaptive evolution of gene expression in nature. Nature 525: 372-375. doi:10.1038/nature15256

Gianuca, A. T., S. A. J. Declerck, M. W. Cadotte, C. Souffreau, T. De Bie, and L. De Meester. 2016a. Integrating trait and phylogenetic distances to assess scale-dependent community assembly processes. Ecography 39: 1-11. doi:10.1111/ ecog.02263

Gianuca, A. T., J. H. Pantel, and L. De Meester. 2016b. Disentangling the effect of body size and phylogenetic distances on zooplankton top-down control of algae. Proc. R. Soc. B Biol. Sci. 283: 1-8. doi:10.1098/rspb.2016.0487

Gienapp, P., C. Teplitsky, J. S. Alho, J. A. Mills, and J. Merilä. 2008. Climate change and evolution: Disentangling environmental and genetic responses. Mol. Ecol. 17: 167-178. doi:10.1111/j.1365-294X.2007.03413.X

Goulden, C. E., L. L. Henry, and A. J. Tessier. 1982. Body size, energy reserves, and competitive ability in three species of Cladocera. Ecology 63: 1780-1789.

Govaert, L., J. H. Pantel, and L. De Meester. 2016. Eco-evolutionary partitioning metrics: Assessing the importance of ecological and evolutionary contributions to population and community change. Ecol. Lett. 19: 839-853. doi:10.1111/ele.12632

Hairston, N. G., Jr., S. P. Ellner, M. A. Geber, T. Yoshida, and J. A. Fox. 2005. Rapid evolution and the convergence of ecological and evolutionary time. Ecol. Lett. 8: 1114-1127. doi:10.1111/j.1461-0248.2005.00812.x

Hendry, A. P., K. M. Gotanda, and E. I. Svensson. 2017. Human influences on evolution, and the ecological and societal consequences. Philos. Trans. R. Soc. B 372: 1-13. doi:10.1098/rstb.2016.0028

Henning-Lucass, N., M. Cordellier, B. Streit, and K. Schwenk. 2016. Phenotypic plasticity in life-history traits of Daphnia galeata in response to temperature-A comparison across clonal lineages separated in time. Ecol. Evol. 6: 881-891. doi:10.1002/ece3.1924

Herrmann, M., K. Schwenk, N. Henning-Lucass, M. Cordellier, and K. Schwenk. 2017. A genotype-Phenotype association approach to reveal thermal adaptation in Daphnia galeata. J. Exp. Zool. 327A: 53-65. doi:10.1002/jez.2070 
Huey, R. B., W. D. Crill, J. G. Kingsolver, and K. E. Weber. 1992. A method for rapid measurement of heat or cold resistance of small insects. Funct. Ecol. 6: 489-494.

Huey, R. B., M. R. Kearney, A. Krockenberger, J. A. M. Holtum, M. Jess, and S. E. Williams. 2012. Predicting organismal vulnerability to climate warming: Roles of behaviour, physiology and adaptation. Phil. Trans. R. Soc. B 367: 1665-1679. doi:10.1098/rstb.2012.0005

IPCC. 2014. Climate change 2014: Synthesis report, p. 151. In Core Writing Team, R. K. Pachauri, and L. A. Meyer [eds.], Contribution of working groups I, II and III to the fifth assessment report of the intergovernmental panel on climate change. Geneva, Switzerland: IPCC.

Jansen, M., A. Coors, J. Vanoverbeke, M. Schepens, P. De Voogt, K. A. C. De Schamphelaere, and L. De Meester. 2015. Experimental evolution reveals high insecticide tolerance in Daphnia inhabiting farmland ponds. Evol. Appl. 8: 442-453. doi:10.1111/eva.12253

Jeppesen, E., and others. 2010. Impacts of climate warming on lake fish community structure and potential effects on ecosystem function. Hydrobiologia 646: 73-90. doi:10.1007/ s10750-010-0171-5

KMI. 2018. Klimatologisch maandoverzicht, zomer 2018.

Korpelainen, H. 1986. The effects of temperature and photoperiod on life history parameters of Daphnia magna (Crustacea: Cladocera). Freshw. Biol. 16: 615-620. doi:10. 1111/j.1365-2427.1986.tb01004.X

Kosten, S., and others. 2012. Warmer climates boost cyanobacterial dominance in shallow lakes. Glob. Chang. Biol. 18: 118-126. doi:10.1111/j.1365-2486.2011.02488.x

Kot, M. 2001. Elements of Mathematical Ecology. Cambridge Univ. Press.

Lampert, W. 2006. Daphnia: Model herbivore, predator and prey. Polish J. Ecol. 54: 607-620.

Lancaster, L. T., G. Morrison, and R. N. Fitt. 2017. Life history trade-offs, the intensity of competition, and coexistence in novel and evolving communities under climate change. Philos. Trans. R. Soc. B 372: 1-10. doi:10.1098/rstb.2016.0046

Lande, R. 2009. Adaptation to an extraordinary environment by evolution of phenotypic plasticity and genetic assimilation. J. Evol. Biol. 22: 1435-1446. doi:10.1111/j.1420-9101. 2009.01754.x

Legendre, P., and L. Legendre. 2012. Numerical Ecology. 24: 3, Elsevier.

Lenth, R. V. 2016. Least-squares means: The R package lsmeans. J. Stat. Softw. 69: 1-33. doi:10.18637/jss. v069.i01

Merilä, J., and A. P. Hendry. 2013. Climate change, adaptation, and phenotypic plasticity: The problem and the evidence. Evol. Appl. 7: 1-14. doi:10.1111/eva.12137

Miner, B. E., L. De Meester, M. E. Pfrender, W. Lampert, and N. G. Hairston Jr. 2012. Linking genes to communities and ecosystems: Daphnia as an ecogenomic model. Proc.
R. Soc. B Biol. Sci. 279: 1873-1882. doi:10.1098/rspb. 2011.2404

Mitchell, S. E., and W. Lampert. 2000. Temperature adaptation in a geographically widespread zooplankter, Daphnia magna. J. Evol. Biol. 13: 371-382. doi:10.1046/j.1420-9101. 2000.00193.x

Moore, M. V. 1996. Consequences of elevated temperatures for zooplankton assemblages in temperate lakes. Arch. Hydrobiol. 135: 289-319.

Nakagawa, S., and I. C. Cuthill. 2007. Effect size, confidence interval and statistical significance: A practical guide for biologists. Biol. Rev. 82: 591-605. doi:10.1111/j.1469185X.2007.00027.x

Neuwald, J. L., and N. Valenzuela. 2011. The lesser known challenge of climate change: Thermal variance and sexreversal in vertebrates with temperature-dependent sex determination. PLoS One 6: 1-9. doi:10.1371/journal.pone. 0018117

Pantel, J. H., C. Duvivier, and L. De Meester. 2015. Rapid local adaptation mediates zooplankton community assembly in experimental mesocosms. Ecol. Lett. 18: 992-1000. doi:10. 1111/ele. 12480

Parmesan, C., and G. Yohe. 2003. A globally coherent fingerprint of climate change impacts across natural systems. Nature 421: 37-42.

Paul, R. J., T. Lamkemeyer, J. Maurer, O. Pinkhaus, R. Pirow, M. Seidl, and B. Zeis. 2004. Thermal acclimation in the microcrustacean Daphnia: A survey of behavioural, physiological and biochemical mechanisms. J. Therm. Biol. 29: 655-662. doi:10.1016/j.jtherbio.2004.08.035

Perkins, S. E. 2015. A review on the scientific understanding of heatwaves-Their measurement, driving mechanisms, and changes at the global scale. Atmos. Res. 164-165: 242-267. doi:10.1016/j.atmosres.2015.05.014

Pfennig, D. W., M. A. Wund, E. C. Snell-Rood, T. Cruickshank, C. D. Schlichting, and A. P. Moczek. 2010. Phenotypic plasticity's impacts on diversification and speciation. Trends Ecol. Evol. 25: 459-467. doi:10.1016/j.tree.2010. 05.006

R Core Team. 2013. R: A language and environment for statistical computing. Foundation for Statistical Computing. Vienna, Austria.

Ranta, E., J. Bengtsson, and J. McManus. 1993. Growth, size and shape of Daphnia longispina, D. magna and D.pulex. Ann. Zool. Fennici 30: 299-311.

Reichstein, M., and others. 2007. Reduction of ecosystem productivity and respiration during the European summer 2003 climate anomaly: A joint flux tower, remote sensing and modelling analysis. Glob. Chang. Biol. 13: 634-651. doi:10.1111/j.1365-2486.2006.01224.x

Scheffers, B., and others. 2016. The broad footprint of climate change from genes to biomes to people. Science 354: 719-731. doi:10.1126/science.aaf7671 
Sheridan, J. A., and D. Bickford. 2011. Shrinking body size as ecological response to climate change. Nat. Clim. Chang. 1: 401-406. doi:10.1038/nclimate1259

Stoks, R., L. Govaert, K. Pauwels, B. Jansen, and L. De Meester. 2016. Resurrecting complexity: The interplay of plasticity and rapid evolution in the multiple trait response to strong changes in predation pressure in the water flea Daphnia magna. Ecol. Lett. 19: 180-190. doi:10.1111/ele.12551

Stott, P. A., D. A. Stone, and M. R. Allen. 2004. Human contribution to the European heatwave of 2003. Nature 432: 610-614. doi:10.1038/nature03089

Tessier, A. J., M. A. Leibold, and J. Tsao. 2000. A fundamental trade-off in resource exploitation by Daphnia and consequences to plankton communities. Ecology 81: 826-841.

Urban, M. C., and others. 2016. Improving the forecast for biodiversity under climate change. Science. 353: 1-9. doi: 10.1126/science.aad8466

Van Doorslaer, W., R. Stoks, I. Swillen, H. Feuchtmayr, D. Atkinson, B. Moss, and L. De Meester. 2010. Experimental thermal microevolution in community-embedded Daphnia populations. Climate Res. 43: 81-89. doi:10.3354/cr00894

Vindenes, Y., E. Edeline, J. Ohlberger, Ø. Langangen, I. J. Winfield, N. C. Stenseth, and L. A. Vøllestad. 2014. Effects of climate change on trait-based dynamics of a top predator in freshwater ecosystems. Am. Nat. 183: 243-256. doi:10. $1086 / 674610$

Wickham, H. 2016. ggplot2: Elegant graphics for Data Analysis. Springer-Verlag. New York.

Winder, M., and D. E. Schindler. 2004. Climate change uncouples trophic interactions in an aquatic ecosystem. Ecology 85: 2100-2106.
Wojtal-Frankiewicz, A. 2012. The effects of global warming on Daphnia spp. population dynamics: A review. Aquat. Ecol. 46: 37-53. doi:10.1007/s10452-011-9380-X

Yampolsky, L. Y., T. M. M. Schaer, and D. Ebert. 2014. Adaptive phenotypic plasticity and local adaptation for temperature tolerance in freshwater zooplankton. Proc. R. Soc. B 281: 1-9. doi:10.1098/rspb.2013.2744

Zeis, B., D. Becker, P. Gerke, M. Koch, and R. J. Paul. 2013. Hypoxia-inducible haemoglobins of Daphnia pulex and their role in the response to acute and chronic temperature increase. Biochim. Biophys. Acta 1834: 1704-1710. doi:10. 1016/j.bbapap.2013.01.036

\section{Acknowledgments}

This work was financially supported by the National Fund for Scientific Research FWO_Flanders project G0B9818N and $1127221 \mathrm{~N}$ and KU Leuven Research Fund project C16/17/002. LG was supported by the University of Zurich Research Priority Program on "Global Change and Biodiversity." We thank Geerts Neyens for the technical support and the students Jelle Dehaen and Lou Van Eupen for their help with the practical work. We thank Mathilde Cordellier and an anonymous reviewer for valuable and insightful suggestions to a previous version of the manuscript.

\section{Conflict of Interest}

None declared.
Submitted 11 February 2020

Revised 31 July 2020

Accepted 03 December 2020

Associate editor: Takehito Yoshida 